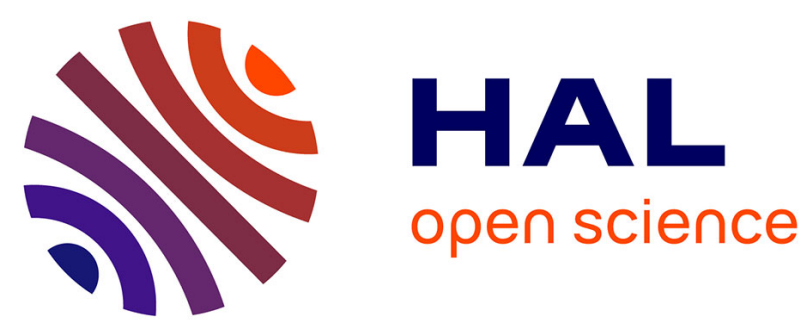

\title{
Comparison of 2 methodologies developed for the determination of residual stresses through X-ray diffraction: application to a textured hep titanium alloy
}

\author{
S. Dufrenoy, T. Chauveau, I. Lemaire-Caron, R. Brenner, B. Bacroix
}

\section{- To cite this version:}

S. Dufrenoy, T. Chauveau, I. Lemaire-Caron, R. Brenner, B. Bacroix. Comparison of 2 methodologies developed for the determination of residual stresses through X-ray diffraction: application to a textured hcp titanium alloy. International Journal of Material Forming, 2018, 11 (3), pp.341-355. 10.1007/s12289-017-1354-7 . hal-01542882

\section{HAL Id: hal-01542882 \\ https://hal.sorbonne-universite.fr/hal-01542882}

Submitted on 20 Jun 2017

HAL is a multi-disciplinary open access archive for the deposit and dissemination of scientific research documents, whether they are published or not. The documents may come from teaching and research institutions in France or abroad, or from public or private research centers.
L'archive ouverte pluridisciplinaire HAL, est destinée au dépôt et à la diffusion de documents scientifiques de niveau recherche, publiés ou non, émanant des établissements d'enseignement et de recherche français ou étrangers, des laboratoires publics ou privés. 


\title{
Comparison of 2 methodologies developed for the determination of residual stresses through
}

$\mathrm{X}$-Ray diffraction: application to a textured hep titanium alloy

\author{
S. Dufrenoy ${ }^{1,2}$, T. Chauveau ${ }^{1}$, I. Lemaire-Caron ${ }^{2}$, R. Brenner $^{3}$, B. Bacroix ${ }^{1 *}$ \\ ${ }^{1}$ LSPM - CNRS, Université Paris 13, 99 av. J.B. Clément, 93430 Villetaneuse, France \\ ${ }^{2}$ QUARTZ, EA7393, Supmeca, 3 rue Fernand Hainaut, 93400 Saint Ouen, France \\ ${ }^{3}$ Institut Jean Le Rond d'Alembert, CNRS - Université Pierre et Marie Curie, UMR 7190, \\ 4 place Jussieu, Paris, France. \\ *Corresponding author, brigitte.bacroix@univ-paris13.fr
}

Keywords: residual stresses, hcp structure, titanium alloys, self-consistent model, X-ray diffraction (XRD).

\begin{abstract}
For polycrystalline materials, the experimental determination of residual stresses neglects the socalled $2^{\text {nd }}$ order fluctuations arising from e.g. plastic or thermal incompatibilities from grain to grain. This constitutes a serious limitation of the classical measurements methods, since these $2^{\text {nd }}$ residual stresses are known to have a major influence on the mechanical behavior of metallic alloys, especially if these are strongly textured.

In the present paper, a new methodology for the treatment of the measured data is described and compared to classical ones. In order to do so, the simulation of a tensile test is performed using a self-consistent elasto-plastic model, in order to constitute a virtual experimental data set. The $1^{\text {st }}$ and $2^{\text {nd }}$ order stresses are extracted from the simulation for various macroscopic stress levels. Two approaches (the classical $\sin ^{2} \psi$ method and a method based on the simultaneous analysis of several X-ray diffraction peaks) are then used to quantify the $1^{\text {st }}$ order stresses from these "experimental" data. It is clearly shown that the method based on multi-peak analysis allows to minimize the error made by neglecting the so-called $2^{\text {nd }}$ order stresses and leads to a better quantitative estimation of the $1^{\text {st }}$ order stresses.
\end{abstract}

\footnotetext{
*This article is dedicated to Prof. José Gracio, with whom I had the pleasure of working several times and discussing about the links between microstructure and mechanical properties of engineering materials. Every moment of exchange gave me the opportunity to discover his availability, creativity and enthusiasm for life and research. For all of us, he has been an example and a source of inspiration and renewed motivation. Surely, he would have actively participated to discussions about residual stresses (B. Bacroix).
} 


\section{Introduction}

Residual stresses are stresses which are retained within a body when no external forces are acting. They arise because of misfits (or incompatibilities) between different regions of the investigated material sample or component, which are a direct consequence of what these have been subjected to. Residual stresses can be classified according to their origin (e.g. [1, 2]), the scale over which they equilibrate (e.g.[3]), or their effect on the macroscopic behavior of a structural piece (e.g.[4]). As they can have beneficial or detrimental effects on the life time of mechanical components, indeed, the three aspects (origin, distribution and influence on mechanical behavior) are important. In the case of polycrystalline materials, the most common origins of the misfits are non-uniform plastic flow, steep thermal gradients or phase transformations. And due to the polycrystalline nature of the materials, these stresses indeed vary from point to point within the microstructure, and thus, several orders of stresses are generally distinguished $[2,5]$ (see below).

In many industrial applications, in which materials and components are subjected to severe and complex thermomechanical treatments (including forming, heat treatment, welding, machining, ...), the origin of the existing residual stresses is usually a combination of several misfit sources (e.g. plasticity and thermal contraction due to rapid cooling [6]), which are not easy to know a priori. The so-called macroscopic $1^{\text {st }}$ order stresses (which are averaged on a quite large volume and thus neglect the underlying microstructure) are of primary importance, since they are indeed the ones which have the greatest influence on the life time of mechanical parts of a structure. They are more and more often determined through X-Ray Diffraction (XRD), and this is why some standards (e.g. [7]) have been developed to define the best protocol to be able to evaluate these stresses from measurements with sufficient quantitative accuracy.

However, all these standards rely on crude assumptions concerning the material, which are rarely verified for metals which present a hexagonal close packed (HCP) crystallographic structure (such as $\mathrm{Zr}, \mathrm{Ti}, \mathrm{Mg}, \ldots .$.$) , and which are widely present e.g. in the aeronautic or energy industries. The$ two main important assumptions which are mentioned in these standards are that (i) the investigated material can be considered isotropic, and (ii) the $2^{\text {nd }}$ order stresses - of inelastic origin - can be neglected. Unfortunately, these two assumptions are rarely met for the above mentioned metals, as well as for dual-phase $\mathrm{Ti}$ alloys with complex microstructures, which are now widely used in the aeronautic industry.

The aim of the present work is thus to propose a modification of the classical methodology, which minimizes the error made on the estimation of the $1^{\text {st }}$ order stress, and thus improves the recommended standard, although it still based on a priori assumptions. In order to validate this new 
method and to compare it to the classical methodology, both are applied to a theoretical case for which the residual stresses are a priori completely known. It consists into the simulation of a tensile test of an anisotropic $\mathrm{Ti}$ sample with a polycrystalline mean field model. The paper is thus organized according to the following outline. The classical definitions of the stresses defined at different scales are first recalled in Section 1; then, the polycrystalline model, tested material and performed simulations are presented in Section 2. The classical standard and proposed methodology are detailed in Section 3. Results obtained with both methods are then presented in Section 4 and compared to those of the polycrystalline model. The paper ends up with some general recommendations about the use of these methods and perspectives.

\section{Definition of the various order stresses for a polycrystalline material}

Let us consider a polycrystalline sample, schematically represented by a 2 dimensional section in Fig. 1. This sample has been e.g. machined on the upper surface (perpendicular to the figure and identified by the direction normal to it, labelled ND), which means that it has been subjected to heterogeneous stress and temperature fields throughout its thickness. As a result, the internal structure and mechanical state within the superficial layer has been modified compared to the lower part of the sample. This induces misfits between various layers, and thus residual stresses. During such a machining operation, plastic strain and heating/cooling are the main processes responsible for the appearance of residual stresses. Due to the polycrystalline structure of the material, plastic deformation, as well as thermal dilatation/contraction, generally varies from grain to grain, producing in turn misfits between grains, i.e. at the intergranular level [8, 9]. And we also know that in such materials, deformation is rarely homogeneous within the grains, resulting in turn into heterogeneities at the intragranular level. The various stresses usually defined in the field of residual stresses are recalled below. As a result of inhomogeneous boundary conditions imposed to the whole sample, the stress state depends on the location. We can thus define stresses at different levels: $\sigma(x)$ the local stress at point $x$ of the sample, $\sigma_{g}$ the average stress for grain $g, \sigma_{V}$ the average stress on a given investigated volume $\mathbf{V}$ containing a large number of grains and finally $\boldsymbol{\sigma}_{S}$ the stress averaged on the whole sample, which can be the imposed stress $\Sigma_{S}^{i m p}$ during a mechanical test. The investigated volume $\mathbf{V}$ can be for example a part of a superficial layer, whose thickness will both depend on the measurement method, as well as the investigated process. It has to be distinguished from the diffracting volume $\Omega$ which is defined latter. In the context of residual stresses, it is common to decompose these various stress as follows 


$$
\begin{gathered}
\boldsymbol{\sigma}(x)=\boldsymbol{\sigma}_{g}+\boldsymbol{\sigma}_{x}^{I I I} \\
\boldsymbol{\sigma}_{g}=\frac{1}{V_{g}} \cdot \int_{V_{g}} \boldsymbol{\sigma}(x) d V=\boldsymbol{\sigma}_{V}+\boldsymbol{\sigma}_{g}^{I I} \\
\boldsymbol{\sigma}_{V}=\frac{1}{V} \cdot \int_{V} \boldsymbol{\sigma}(x) d V=\boldsymbol{\sigma}_{S}+\boldsymbol{\sigma}_{V}^{I} \\
\boldsymbol{\sigma}_{S}=\frac{1}{V_{S}} \cdot \int_{V_{S}} \boldsymbol{\sigma}(x) d V=\Sigma_{S}^{i m p}
\end{gathered}
$$

We see that $\boldsymbol{\sigma}_{x}^{I I I}, \boldsymbol{\sigma}_{g}^{I I}, \boldsymbol{\sigma}_{V}^{I}$ are fluctuations around average values, which are classically called $3^{\text {rd }}, 2^{\text {nd }}$ and $1^{\text {st }}$ order stresses respectively. When the whole sample is unloaded, then $\Sigma^{i m p}=\sigma_{S}=$ 0 and $\sigma_{V}=\sigma_{V}^{I}$. In this case, $\sigma_{V}^{I}$ correspond to the $1^{\text {st }}$ order residual stress tensor (averaged on the investigated volume) that we want to evaluate. The $2^{\text {nd }}$ order stresses, $\boldsymbol{\sigma}_{g}^{I I}$, which correspond to fluctuations within the grains around $\boldsymbol{\sigma}_{V}^{I}$, are then the $2^{\text {nd }}$ order residual stresses. If the whole sample has been deformed homogeneously, then, in the unloaded state, we necessarily have

$\boldsymbol{\sigma}_{V}=\boldsymbol{\sigma}_{V}^{I}=0$ and thus $\boldsymbol{\sigma}_{g}=\boldsymbol{\sigma}_{g}^{I I}$. The investigated volume can be considered in this case to be a Representative Volume Element (RVE) for the material. We will only have $2^{\text {nd }}$ order residual stresses in this case. In what follows, we will neglect the intragranular stress fluctuations $\left(\boldsymbol{\sigma}_{x}^{I I I}\right)$ and consider only $1^{\text {st }}$ and $2^{\text {nd }}$ order stresses.

\section{Simulation of a tensile test}

We start simulating a tensile test in a textured polycrystalline sample (supposed to be initially stress free), in order to estimate the stress distribution within the polycrystalline sample for various macroscopic stress levels. In other words, we create an artificial "experimental" data set, for which the $2^{\text {nd }}$ order residual stresses will be due to plasticity only. The considered material is commercially pure titanium (grade 2), whose initial texture in presented in Fig. 2. The texture is typical from rolled and annealed titanium, and is composed of two main texture components, which are the socalled tilted $\{0001\}\langle 11 \overline{2} 0\rangle$ and tilted $\{0001\}\langle 1 \overline{1} 00\rangle$ orientations [10]. This texture statistically respects the orthotropic symmetry arising from the rolling process and is far from isotropy (the maximum is larger than 6 for the $\{0002\}$ pole figure). The Orientation Distribution Function is calculated from pole figures using the LaboTex ${ }^{\circledR}$ Software and a dataset of 50000 orientations is extracted, to represent the texture for further calculations. 
The original elasto-plastic SC model, initially developed by Kröner [11] for the case of elasticity, and further extended to the treatment of thermo-elasto-plasticity by Hutchinson [12] and Turner et al. [6] has been used here, since it has been shown to be well adapted to the calculation of $1^{\text {st }}$ order stresses arising from plasticity or thermal treatment for HCP materials [13, 14]. Compared to other polycrystalline models used un the context of residual stresses (see e.g. $[15,16]$ ), it has also the advantage of being solely based on mechanical interactions between one grain and the surrounding material, without the addition of any fitting parameter. It is used here to simulate a tensile test along $\mathrm{RD}$ up to $5 \%$ total strain, i.e. in elasto-plasticity (thermal effects are not considered here). It is then possible to extract the stresses $\boldsymbol{\sigma}_{V}^{I}$ and $\boldsymbol{\sigma}_{g}^{I I}$ within each grain of the material at various stages of the loading process. The use of this model is based on the following procedure:

(i) The material is considered to be heterogeneous, i.e. composed of a discrete set of grains of various orientations composing the texture; here, the sole consideration of the distribution of orientations is taken into account and not their position;

(ii) The simulation is performed on a so-called Representative Volume Element (RVE), on which the boundary conditions of the simulated test are imposed;

(iii) The localization step allows establishing relationships between the macroscopic quantities (at the level of the RVE) and the microscopic ones (at the level of each grain) and the knowledge of the local constitutive law allows getting the unknown macroscopic quantities through the homogenization step.

Only the principal equations relating stress and strain tensors (or stress and strain rate tensors) will be recalled below, and the reader is referred to the work of Turner, Tome et al. $[4,6,17,18]$ for further details concerning this model. It is important to note that during plastic deformation, the model not only allows the quantifications of these tensors but also to calculate the grain shapes and texture evolutions due to slip on several systems within each grain. For the performed simulations, because of the limited amount of imposed strain, the grain shape will be considered to be constant, which reduces the computing time, but texture evolution, although expected to be limited, will be considered.

If the model is used in the elastic range, then the local law relating elastic strain $\boldsymbol{\varepsilon}_{g}$ and stress $\boldsymbol{\sigma}_{\boldsymbol{g}}$ at the level of the grains reads

$$
\begin{aligned}
& \boldsymbol{\sigma}_{g}=\boldsymbol{C}_{g}: \boldsymbol{\varepsilon}_{g} \\
& \boldsymbol{\varepsilon}_{g}=\boldsymbol{S}_{g}: \boldsymbol{\sigma}_{g}
\end{aligned}
$$

With $\boldsymbol{C}_{g}$ and $\boldsymbol{S}_{g}$ the $4^{\text {th }}$ order elastic stiffness and compliance tensors respectively. 
The localization step allows to write

$$
\begin{aligned}
& \boldsymbol{\sigma}_{g}=\boldsymbol{B}_{g}: \boldsymbol{\Sigma} \\
& \boldsymbol{\varepsilon}_{g}=\boldsymbol{A}_{g}: \mathbf{E}
\end{aligned}
$$

where $\boldsymbol{\Sigma}$ and $\mathbf{E}$ are the macroscopic quantities (at the level of the RVE) and $\boldsymbol{B}_{g}$ and $\boldsymbol{A}_{g}$ are the $4^{\text {th }}$ order tensors of stress concentration or strain localization respectively. The average of these $4^{\text {th }}$ order tensors over the grains contained within the RVE (represented below by the symbol $\langle.\rangle_{R V E}$ ) is equal to the identity tensor, since by definition we have

$$
\left\langle\boldsymbol{\sigma}_{g}\right\rangle_{R V E}=\boldsymbol{\Sigma} \text { and }\left\langle\boldsymbol{\varepsilon}_{g}\right\rangle_{R V E}=\mathbf{E}
$$

We can then write macroscopic constitute laws - a priori unknown - of the same form as the local ones,

$$
\begin{aligned}
& \boldsymbol{\Sigma}=\widetilde{\boldsymbol{C}}: \mathbf{E} \\
& \mathbf{E}=\tilde{\boldsymbol{S}}: \boldsymbol{\Sigma}
\end{aligned}
$$

where $\widetilde{\boldsymbol{C}}$ and $\tilde{\boldsymbol{S}}$ are called the $4^{\text {th }}$ order macroscopic stiffness and compliance tensors.

Then, if we assume that each grain is a homogeneous ellipsoidal inclusion embedded into an infinite medium, the average localization strain tensor reads

$$
\boldsymbol{A}_{g}=\left[\boldsymbol{I}+\boldsymbol{S}^{E}: \widetilde{\boldsymbol{C}}^{-1}:\left(\boldsymbol{C}_{g}-\widetilde{\boldsymbol{C}}\right)\right]^{-1}
$$

with $\boldsymbol{S}^{E}$, the Eshelby tensor, which depends on the shape of the grain $g$ and on the infinite medium. This equation leads to the determination of all local and global quantities from macroscopic imposed boundary equations.

Now, the extension of this model to the case of elasto-plasticity (EP) requires considering incremental laws. The crystal plasticity law assumes that plastic strain takes places within each grain $g$ by slip on specific slip systems, which are potentially active when

$$
\boldsymbol{\sigma}_{g}: \boldsymbol{R}^{s}=\tau_{c}^{s} \text { with } \dot{\gamma}^{s} \geq 0
$$

$\boldsymbol{R}^{s}$ is the Schmid tensor, $\tau_{c}^{S}$ the critical resolved shear stress and $\dot{\gamma}^{s}$ the shear rate for system $s$. The components of the Schmid tensor are classically defined by 


$$
R_{i j}^{S}=\frac{1}{2}\left(m_{i}^{S} n_{j}^{S}+m_{j}^{S} n_{i}^{S}\right)
$$

with $\vec{m}^{s}$ and $\vec{n}^{s}$ being unit vectors representing the slip plane normal and slip direction of system $s$ and $m_{i}^{S}$ and $n_{j}^{S}$ being the individual components of these vectors.

By decomposing the total strain into the sum of the elastic and plastic parts, we get

$$
\dot{\boldsymbol{\varepsilon}}_{g}=\boldsymbol{S}_{g}: \dot{\boldsymbol{\sigma}}_{g}+\sum_{p} R^{p} \dot{\gamma}^{p}=\boldsymbol{M}_{g}: \dot{\boldsymbol{\sigma}}_{g}
$$

The Hooke's law which relates the stress rate to the elastic strain rate now writes

$$
\dot{\boldsymbol{\sigma}}_{g}=\boldsymbol{C}_{g}:\left(\dot{\boldsymbol{\varepsilon}}_{g}-\sum_{p} R^{p} \dot{\gamma}^{p}\right)=\boldsymbol{L}_{g}: \dot{\boldsymbol{\varepsilon}}_{g}
$$

The previous elastic stiffness $\boldsymbol{C}_{g}$ and compliance $\boldsymbol{S}_{g}$ tensors are then replaced by tangent $\boldsymbol{L}_{g}$ and $\boldsymbol{M}_{g}$ modulus tensors. Again we can write macroscopic laws

$$
\begin{aligned}
\dot{\Sigma} & =\widetilde{L}: \dot{\mathbf{E}} \\
\dot{\mathbf{E}} & =\widetilde{M}: \dot{\Sigma}
\end{aligned}
$$

in terms of strain and stress rates, in which $\widetilde{\boldsymbol{L}}$ and $\widetilde{\boldsymbol{M}}$ are called the $4^{\text {th }}$ order macroscopic effective moduli.

After Yoshida et al. [19], we consider the following saturating hardening law for the slip systems

$$
\begin{gathered}
\dot{\tau}_{c}^{S}=\sum_{p} h^{s p} \dot{\gamma}^{p} \\
\text { with } h^{s p}=\left[q+(1-q) \delta^{s p}\right] h_{0}\left(1+\frac{h_{0} \gamma_{a}}{\tau_{r e f} n}\right)^{n-1}
\end{gathered}
$$

In this equation, $\gamma_{a}=\int_{0}^{t} \sum_{s}\left|\dot{\gamma}^{s}\right| d t$ is the total strain, $q, h_{0}$ and $n$ are hardening parameters and $\tau_{r e f}$ is the reference critical shear stress of the so-called easy glide systems. The selected slip systems families and associated parameters, which have been identified on experimental tensile curves obtained by Benmhenni et al. for commercially pure titanium [20], are listed in Table 1.

The single crystal elastic constants are taken equal to: $C_{11}=162.4 \mathrm{GPa}, C_{12}=92 \mathrm{GPa}$, $C_{13}=69 \mathrm{GPa}, C_{33}=180.7 \mathrm{GPa}, C_{44}=46.7 \mathrm{GPa}$ [21]. It is worth mentioning that the HCP single crystal presents transverse isotropy, which means that the Young modulus in tension is constant in 
the basal plane. It is also seen in Fig. 3 (left) that it varies from $10.4 \mathrm{GPa}$ in the basal plane to 14.4 $\mathrm{GPa}$ along the $<\mathrm{c}>$ axis. In other words, the single crystal elastic anisotropy is limited but not completely negligible. When the SC model is used to calculate the macroscopic Young's modulus of the textured polycrystal as a function of the tensile direction, the range is still reduced to 10.8 to 12.4 GPa but small variations are now observed within the (X,Y) plane (Fig. 3 right). Thus, we can say that the material is macroscopically moderately elastically anisotropic.

Now, the boundary conditions imposed to the RVE to simulate a tensile test are the following

$$
\dot{\mathrm{E}}=\left(\begin{array}{ccc}
\dot{\mathrm{E}}_{11} & ? & ? \\
? & ? & ? \\
? & ? & ?
\end{array}\right) \text { and } \dot{\Sigma}=\left(\begin{array}{ccc}
? & 0 & 0 \\
0 & 0 & 0 \\
0 & 0 & 0
\end{array}\right)
$$

In other words, we solely impose a macroscopic uniaxial stress tensor together with the magnitude of the tensile strain rate $\dot{\mathrm{E}}_{11}$. The simulated curve is shown in Fig. 4 (left). The principal strains (width and thickness strains) are also presented in Fig. 4 (right). It is worth noting that this plot highlights the plastic anisotropy of the material, since these two strains would be equal for an isotropic sample. It is seen on this curve, that these two strains are indeed almost equal at the very beginning of the deformation, i.e. in the elastic range, as expected from the calculation of the Young's modulus in this range.

Apart from the initial state characterized by the point labelled 0 and which corresponds to zero stress everywhere in the RVE, 4 points have been identified along the curve, at which $1^{\text {st }}$ and $2^{\text {nd }}$ order stresses will be calculated. For that purpose, the RVE will represent the investigated volume described above (i.e. a part of a larger sample), subjected to the calculated macroscopic stress state, which will thus be equivalent to a residual stress state $\Sigma_{V}=\boldsymbol{\sigma}_{V}^{I}$. It is worth noting that the classical situation in which residual stresses are usually measured corresponds actually to point 4 , i.e. a state at which residual stresses due to an heterogeneous plastic strain field have been previously introduced into the material at the level of each grain $\left(\boldsymbol{\sigma}_{g E P}^{I I}\right)$ as well as at the level of the RVE $\left(\boldsymbol{\sigma}_{V}^{I}\right)$, which thus remains elastically loaded, although being a part of an unloaded sample. For each of these points, the macroscopic stress as well as the distribution of stresses inside the grains will be extracted from the calculations. For each of these points, we can write

At point 0:

$$
\boldsymbol{\sigma}_{g}=\boldsymbol{\sigma}_{V}^{I}=0
$$

At point 1(elastic range):

$$
\boldsymbol{\sigma}_{V}^{I} \neq 0 \text { and } \boldsymbol{\sigma}_{g}=\boldsymbol{B}_{g}: \boldsymbol{\sigma}_{V}^{I}
$$

with $\boldsymbol{B}_{g}$ the elastic stress concentration tensor. 
At point 2

(elasto-plastic range):

$\boldsymbol{\sigma}_{V}^{I} \neq 0$ but no simple relationship exists between global and local stresses, since the final stress state depends on the whole strain path; from the definitions given in Eq. 1, we can write

$$
\boldsymbol{\sigma}_{g}=\boldsymbol{\sigma}_{V}^{I}+\boldsymbol{\sigma}_{g E P}^{I I}
$$

where $\sigma_{g E P}^{I I}$ are intergranular fluctuations around the average value $\boldsymbol{\sigma}_{V}^{I}$ due to the elasto-plastic strain. This can be rewritten as

$$
\boldsymbol{\sigma}_{g}=\boldsymbol{B}_{g}: \boldsymbol{\sigma}_{V}^{I}+\left(I-\boldsymbol{B}_{g}\right): \boldsymbol{\sigma}_{V}^{I}+\boldsymbol{\sigma}_{g E P}^{I I}
$$

At point 3 (unloading):

$$
\boldsymbol{\sigma}_{V}^{I}=0 \text { and } \boldsymbol{\sigma}_{g}=\boldsymbol{\sigma}_{g E P}^{I I}
$$

At point 4 (elastic range):

$$
\boldsymbol{\sigma}_{g}=\boldsymbol{B}_{g}: \boldsymbol{\sigma}_{V}^{I}+\boldsymbol{\sigma}_{g E P}^{I I}
$$

Thus, because of the rewriting of Eq.14c, at each step of the loading path, the stress state within grain $g$, can be expressed by the general expression

$$
\boldsymbol{\sigma}_{g}=\boldsymbol{B}_{g}: \boldsymbol{\sigma}_{V}^{I}+\boldsymbol{\sigma}_{g N E}^{I I}
$$

i.e., the sum of two terms, the $1^{\text {st }}$ one associated with the macroscopic $1^{\text {st }}$ order stress state $\sigma_{V}^{I}$, and the second one associated with the fluctuations - of Non Elastic (NE) origin - arising from the overall thermomechanical history of the sample and related in the present case solely to elastoplastic incompatibilities (EP). However, in the context of stress measurements associated with severe processes such as machining or additive manufacturing, plasticity generally occurs concurrently with thermal expansion or phase transformations, and the term $\boldsymbol{\sigma}_{g N E}^{I I}$ is then a consequence of all these processes occurring heterogeneously within the material. However, unlike in the case of elasto-plasticity, the quantitative prediction of this term is still a challenge, which necessitates complex numerical tools (see e.g. [16, 22]). In the present case, reduced to elastoplasticity, $\boldsymbol{\sigma}_{V}^{I}$, as well as $\boldsymbol{\sigma}_{g E P}^{I I}$ (and thus $\boldsymbol{\sigma}_{g N E}^{I I}$ ) can be extracted from our simulations, for all the points highlighted on the curve (in Fig. 4). Due to the definition of the $2^{\text {nd }}$ order stresses, we also have $\left\langle\boldsymbol{\sigma}_{g E P}^{I I}\right\rangle_{R V E}=0 \Rightarrow\left\langle\boldsymbol{\sigma}_{g N E}^{I I}\right\rangle_{R V E}=0$.

\section{Classical method for the determination of residual stresses}


As already mentioned, residual stresses are necessarily associated with residual elastic strains ensuring the compatibility of the total strain. But, as seen previously from the above detailed equations, they may simultaneously depend on elastic, thermal and plastic properties of the material. Diffraction (of neutrons or X-rays) is now a widely-used technique to measure these elastic strains. It is also worth noting that this technique is ideally suited for comparison with mean - field models (such as the one described above), since both methodologies allow the determination of the global response of a large number of grains, without explicit account of their position within the sample. Several types of experimental set-up exist to measure these strains, from classical laboratory devices to neutron sources which allow the characterization of large volumes [23] or third generation synchrotron sources such as the European Synchrotron Radiation Facility (ESRF) which provide intense beams of high energy X-rays that are much more penetrating than characteristic laboratory X-ray devices [24]. The experimental devices and set-up depend also to a certain extent whether we need to examine thin films or thick samples [25]. Recently, a new device has been developed, which allows a rapid determination of a 2D X-ray image, composed of several peaks [26]. Whatever the precision, penetration depth and experimental set up, all these devices are based on the same type of analysis, which relies on the measurement of elastic strains, averaged, not on the whole investigated volume, but on a so-called diffracting volume $\Omega$ (see Fig. 1), only composed, for a given diffracting direction, of all grains in diffracting orientation.

For a given X-ray source of wavelength $\lambda$, and an imposed diffraction vector $\boldsymbol{K}$, characterized by the plane indices $\{h k l\}$ and the two angles $\psi$ and $\phi$ with respect to the sample reference frame (see Fig. 5), it is possible to measure the actual lattice parameter of the screened plane family, averaged on all grains in diffraction position, i.e. those composing the diffracting volume $\Omega$. The Bragg's law reads in this case

$$
2\left\langle d_{\{h k l\}}\right\rangle_{\Omega} \sin \theta_{\{h k l\}}=n \lambda
$$

with $\theta_{\{h k l\}}$ the Bragg angle associated with the plane family $\{h k l\}$ and the X-ray source, and $\left\langle d_{\{h k l\}}\right\rangle_{\Omega}$ the lattice parameter in the direction of the diffraction vector averaged on all diffracting grains. From this, we can get the elastic strain, again averaged on $\Omega$, provided that the initial lattice parameter (under zero stress) $d_{0\{h k l\}}$ is known. This average elastic strain reads

$$
\left\langle\varepsilon_{\phi \psi}\right\rangle_{\Omega \text { mes. }}=\frac{\left\langle d_{\{h k l\}}\right\rangle_{\Omega}-d_{0\{h k l\}}}{d_{0\{h k l\}}}
$$

This strain (which indeed constitutes one single component of the strain tensor) is measured along the direction characterized by the direction $(\psi, \phi)$, i.e. in the reference frame linked to diffraction. 
We denote by A the rotation matrix between the two references frames (sample and diffraction), such that

$$
\boldsymbol{X}_{i j}^{\prime}=A_{i k} A_{j l} \boldsymbol{X}_{k l}
$$

where $\boldsymbol{X}_{k l}$ and $\boldsymbol{X}_{i j}^{\prime}$ are the components of a given tensor, expressed in the reference frames linked to the sample and to diffraction respectively. This rotation matrix reads

$$
A=\left(\begin{array}{ccc}
\cos \phi \cos \psi & \sin \phi \cos \psi & -\sin \psi \\
-\sin \phi & \cos \phi & 0 \\
\cos \phi \sin \psi & \sin \phi \sin \psi & \cos \psi
\end{array}\right)
$$

And we can thus write

$$
\left\langle\varepsilon_{\phi \psi}\right\rangle_{\Omega \text { mes. }}=\left\langle\varepsilon_{33}{ }^{\prime}\right\rangle_{\Omega \text { mes. }}
$$

Now, in order to see how this quantity is related to the residual stresses, we first suppose that the measured elastic strains are the sole consequence of a prior heterogeneous elasto-plastic strain field within the sample, similar to the one simulated above with the SC model. We can then calculate the same quantity with the model as the one measured by first expressing the relationship between calculated elastic strain and stress tensors for one grain $g$, in the diffraction reference frame (by using Eq. 15)

$$
\boldsymbol{\varepsilon}_{\text {gcal. }}^{\prime}=\boldsymbol{S}_{g}^{\prime}: \boldsymbol{\sigma}_{\text {gcal. }}^{\prime}=\boldsymbol{S}_{g}^{\prime}:\left(\boldsymbol{B}_{g}^{\prime}: \boldsymbol{\sigma}_{V \text { cal. }}^{\prime I}+\boldsymbol{\sigma}_{g N E \text { cal. }}^{\prime I I}\right)^{\dagger}
$$

then by averaging this elastic strain on all diffracting grains

$$
\left\langle\boldsymbol{\varepsilon}^{\prime}{ }_{\boldsymbol{g}}\right\rangle_{\Omega \text { cal. }}=\left\langle\boldsymbol{S}_{g}^{\prime}: \boldsymbol{B}_{g}^{\prime}\right\rangle_{\Omega}: \boldsymbol{\sigma}_{V \text { cal. }}^{\prime I}+\left\langle\boldsymbol{S}_{g}^{\prime}: \boldsymbol{\sigma}_{g N E \text { cal. }}^{\prime I I}\right\rangle_{\Omega}
$$

and finally by extracting the component of interest

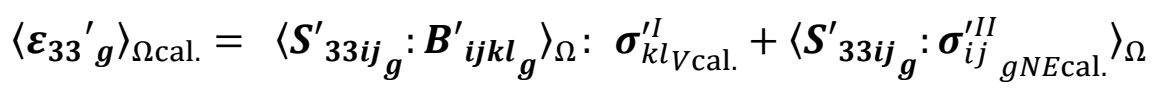

Now, if we want the $1^{\text {st }}$ order residual stress $\boldsymbol{\sigma}_{V}^{I}$ to be expressed in the reference frame linked to the sample, we can write

\footnotetext{
${ }^{\dagger}$ Of course, in this equation, as in Eqs. 22 to 24, the stress tensors are not the only calculated quantities: the tensor $\boldsymbol{B}_{g}{ }_{g}$ is also calculated with the model.
} 


$$
\begin{gathered}
\left\langle\varepsilon_{\phi \psi}\right\rangle_{\Omega \text { cal. }}=\left\langle\boldsymbol{\varepsilon}_{\mathbf{3 3} \boldsymbol{g}}\right\rangle_{\Omega \text { cal. }} \\
=\left\langle\boldsymbol{S}_{\mathbf{3 3 i j}_{\boldsymbol{g}}}^{\prime}: \boldsymbol{B}^{\prime}{ }_{\boldsymbol{i j k \boldsymbol { k }} \boldsymbol{g}}\right\rangle_{\Omega}: A_{k m} A_{l n} \boldsymbol{\sigma}_{m n_{V \text { cal. }}}^{I}+\left\langle\boldsymbol{S}_{\mathbf{3 3 i j}_{\boldsymbol{g}}}^{\prime}: \boldsymbol{\sigma}_{i j}^{\prime I I}{ }_{\text {gNEcal. }}\right\rangle_{\Omega}
\end{gathered}
$$

or more globally

$$
\left\langle\varepsilon_{\phi \psi}\right\rangle_{\Omega \text { cal. }}=\left\langle\varepsilon_{\phi \psi}\right\rangle_{\Omega \text { cal. }}^{I}+\left\langle\varepsilon_{\phi \psi}\right\rangle_{\Omega \text { cal }}^{I I}
$$

Assuming now that the same relationship holds between the measured strain and the $1^{\text {st }}$ and $2^{\text {nd }}$ order stress tensors underwent by the sample and constituting grains, a priori unknown, we can also write

$$
\begin{aligned}
& \left\langle\varepsilon_{\phi \psi}\right\rangle_{\Omega \text { mes. }} \cong\left\langle\varepsilon_{\phi \psi}\right\rangle_{\Omega \text { mod. }}
\end{aligned}
$$

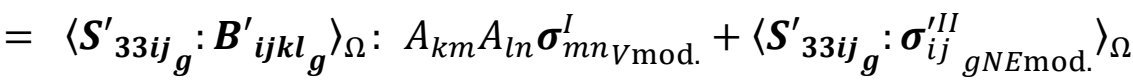

Or more simply

$$
\left\langle\varepsilon_{\phi \psi}\right\rangle_{\Omega \text { mes. }} \cong\left\langle\varepsilon_{\phi \psi}\right\rangle_{\Omega \text { mod. }}=\left\langle\varepsilon_{\phi \psi}\right\rangle_{\Omega \text { mod. }}^{I}+\left\langle\varepsilon_{\phi \psi}\right\rangle_{\Omega \text { mod. }}^{I I}
$$

Equation (25a) thus relates a quantity which can be measured by X-ray diffraction $\left\langle\varepsilon_{33}{ }^{\prime}\right\rangle_{\Omega \text { mes. }}$ with (i) the $1^{\text {st }}$ and $2^{\text {nd }}$ order stress tensors that we want evaluate, $\boldsymbol{\sigma}_{V}^{I}$ and $\boldsymbol{\sigma}_{g N E}^{\prime I I}$ (of various origins) and (ii) some additional unknown tensor $\boldsymbol{B}^{\prime g}$ (which describes the sole elastic behavior of the material). The matrix $A$ is defined experimentally and the stress components $\boldsymbol{S}_{{ }_{33 i \boldsymbol{j}}}$ derived from the elastic constants of the single crystal are usually taken from the literature.

Unfortunately, in the general case of an anisotropic and strongly textured material, it is difficult to get a quantitative estimation of all stress tensors from the sole measurements (there are indeed too many unknowns in both terms of Eq. (25a)); this difficulty can be overcome by making some additional assumptions based on the following considerations:

(i) The second term $\left\langle\varepsilon_{\phi \psi}\right\rangle_{\Omega \text { mod. }}^{I I}=\left\langle\boldsymbol{S}_{\mathbf{3 3 i j}_{\boldsymbol{g}}}^{\prime}: \boldsymbol{\sigma}_{i j}^{\prime I I}{ }_{\text {gNEmod. }}\right\rangle_{\Omega}$ depends on the whole thermomechanical history of the material and thus the only way to get a precise estimate of all included $2^{\text {nd }}$ order stress tensors is to simulate this history with sufficient precision, which is, as already mentioned, an extremely difficult task, still out of reach for many complex processes, such as e.g. welding; however, as these $2^{\text {nd }}$ order stresses arise from fluctuations

\footnotetext{
${ }^{\ddagger}$ We distinguish here the calculated (calc.) quantities from the modelled (mod.) ones: the first ones refer to the predicted quantities using the SC model; the second ones refer the approximated expression we will fit on the measurement points. This expression can be more or less simple and may also need the use of the SC model, as seen below.
} 
around average values, we assume that they can be neglected ( $1^{\text {st }}$ assumption) and concentrate on the determination of the $1^{\text {st }}$ order stress tensor. In fact, in some studies, the second term is even completely omitted, without any justification [25]. Eq. (25) can then be re-written as:

$\left\langle\varepsilon_{\phi \psi}\right\rangle_{\Omega \text { mod. }}=\left\langle\boldsymbol{S}_{\mathbf{3 3 i j}_{\boldsymbol{g}}}^{\prime}: \boldsymbol{B}^{\prime}{ }_{\boldsymbol{i j k \boldsymbol { k }} \boldsymbol{g}_{\boldsymbol{g}}}\right\rangle_{\Omega}: A_{k m} A_{l n} \boldsymbol{\sigma}_{m n_{V \bmod .}}^{I}=\boldsymbol{S}_{33 i j}^{e q} A_{k m} A_{l n} \boldsymbol{\sigma}_{m n_{V \bmod }}^{I}$

In this expression, the matrix $A$ depends solely on the measurement direction but the tensor $\boldsymbol{S}^{\prime e q}$ depends in quite a complex way on the single crystal elastic constants as well as on the diffraction volume (and thus the texture of the material). If we want to avoid requiring to a model to assess it, we can make the following assumptions:

(ii) In the general case, the stress tensor $\boldsymbol{\sigma}_{V}^{I}$ has 6 independent components, but it often reduces to 3 , since it is usually considered that the stress state measured on the surface of the material is a plane stress state, because of the free surface ( $2^{\text {nd }}$ assumption);

(iii) If the material can be considered to be elastically isotropic ( $3^{\text {rd }}$ assumption), the $\boldsymbol{S}^{\text {eq }}$ tensor does not depend anymore on the diffracting plane and volume, but solely on the macroscopic elastic constants which are known a priori (called $S_{1 M}$ and $S_{2 M}$ in Eq. (27) below, defined from $E$ and $v$, the macroscopic Young Modulus and Poisson ratio) [7]. In this case, Eq. (26) reduces to the well-known $\sin ^{2} \psi$ linear fit (because $\left\langle\varepsilon_{\phi \psi}\right\rangle_{\Omega}$ becomes linear as a function of $\sin ^{2} \psi$, see Eqs (27) and (28)) and only one peak and three $\phi$ values (to extract the 3 stress components) are needed. For hexagonal materials, the $\{12 \overline{3} 3\}$ peak is generally recommended, because of the high multiplicity of this crystallographic plane family.

$$
\begin{aligned}
& \left\langle\varepsilon_{\phi \psi}\right\rangle_{\Omega \text { mod. }}=\frac{1+v}{E}\left(\boldsymbol{\sigma}_{11_{V \text { mod. }}^{I}} \cos ^{2} \phi+\boldsymbol{\sigma}_{12_{V \text { mod. }}^{I}} \sin 2 \phi+\boldsymbol{\sigma}_{22_{V \text { mod. }}^{I}}^{I} \sin ^{2} \phi\right) \sin ^{2} \psi \\
& -\frac{v}{E}\left(\boldsymbol{\sigma}_{11_{V \text { mod. }}^{I}}+\boldsymbol{\sigma}_{22 \text { Vmod. }}^{I}\right)=\frac{1}{2} S_{2 M} \cdot \sigma_{\phi \text { mod. }} \cdot \sin ^{2} \psi+S_{1 M} \operatorname{Tr} \sigma_{\text {mod. }} \\
& S_{1 M}=-v / E \quad \text { and } \quad \frac{1}{2} S_{2 M}=\frac{1+v}{E} \\
& \sigma_{\phi \text { mod. }}=\boldsymbol{\sigma}_{11_{V \text { mod. }}^{I}}^{I} \cos ^{2} \phi+\boldsymbol{\sigma}_{12_{V \text { mod. }}^{I}}^{I} \sin 2 \phi+\boldsymbol{\sigma}_{22_{V \text { mod. }}^{I}}^{I} \sin ^{2} \phi
\end{aligned}
$$

with

(iv) In any other case, the $\boldsymbol{S}^{\prime e q}$ tensor has to be calculated with a polycrystalline model describing the elastic response of the material, like e.g. the one used above to simulate the tensile curve $[8,11]$, and the modelled average strain then reads 


$$
\left\langle\varepsilon_{\phi \psi}\right\rangle_{\Omega \text { mod. }}=\frac{1}{2} \boldsymbol{S}_{\Omega 1}^{\prime e q} \cdot \sigma_{\phi \text { mod. }} \cdot \sin ^{2} \psi+\boldsymbol{S}_{\Omega 2}^{\prime e q} \operatorname{Tr} \sigma_{\text {mod. }}
$$

The terms $\boldsymbol{S}_{\Omega 1}^{e q}$ and $\boldsymbol{S}_{\Omega 1}^{e q}$ - called the Diffraction Elastic Constants (DEC) - which now depend on the diffracting plane as well as on diffracting volume $\Omega$ (relative to the texture of the material) replace the macroscopic quantities, and can also be calculated with the SC model in it elastic version. It is worth mentioning though that, if the SC model has proven its excellent predictive capacity in elasticity, some other models, such as Voigt, Reuss (or the average of the two previous ones, also called Hill estimation) can also be selected since they provide explicit relations for the macroscopic Hooke's law and are thus much simpler to use while still providing an acceptable accuracy [27].

The 3 macrostress components are then estimated by the least square method, i.e. by minimizing the following expression, in which the sum is generally performed - for one selected diffraction peak - on all measured points $(i)$, associated with varying angles $(\psi, \phi)$.

$$
\sum_{i}\left\{\left\langle\varepsilon_{\phi \psi}\right\rangle_{\Omega \text { mes. }}-\left\langle\varepsilon_{\phi \psi}\right\rangle_{\Omega \text { mod. }}\right\}^{2}=\operatorname{Min}
$$

In this equation, $\left\langle\varepsilon_{\phi \psi}\right\rangle_{\Omega \text { mod. }}$ is represented either by Eq. 27 if we assume elastic isotropy or by Eq. 28 if we do not assume elastic isotropy. In this case, the material can further be untextured (i.e. macroscopically isotropic) or textured. In the first case, it is easy to show that the average elastic strain is again linear with respect to $\sin ^{2} \psi$, but with a slope which varies with the diffraction plane. In any other case, the variation generally becomes non linear. In most cases, the minimization is realized on a series of measurements performed for one single peak, but some recent procedures rely on multiple peak minimization [28, 29]. It has also been suggested by the French standard that the DEC can also be experimentally determined if the material is single-phased using 4-points bending test with XRD, but it is rarely done in practice $[7,30]$.

For industrial applications, the material is most often considered elastically isotropic, which suppresses the necessity to go through a complex calculation. We now consider our predicted data as a virtual experimental data set and evaluate the impact of the proposed simplifying assumptions on the precision obtained on the quantitative estimate of the sole $1^{\text {st }}$ order macroscopic stress.

\section{Results}


For each of the 4 selected points along the predicted tensile curve, macroscopic elastic strains $\left\langle\varepsilon_{\phi \psi}\right\rangle_{\Omega \text { cal. }}$ have been calculated with the elasto-plastic model for 2 diffracting peaks $\{12 \overline{3} 3\}$ and $\{0006\}$ (the $1^{\text {st }}$ one associated with a high multiplicity and the $2^{\text {nd }}$ with a very low multiplicity) and several values of $\psi$ and 2 values of $\phi$. For these calculations, both elastic and textural anisotropies have been taken into account and the averaging have been made on all grains in diffracting position, with an additional spread of $5^{\circ}$ around them. These calculated elastic strains are represented in Fig. 6 to 9 and the associated diffracting volumes (evaluated at 0 strain) are shown in Fig. 10. For points 2 and 4, for which the elastic strains contain two components (see Eq. (24b)), these two components have been also represented separately.

These figures call for the following comments:

(i) It is interesting to note, that for both peaks, the predicted evaluations of the $1^{\text {st }}$ order strain component $\left\langle\varepsilon_{\phi \psi}\right\rangle_{\Omega \text { cal. }}^{I}$ appear quasi-linear for the 4 points, in spite of the fact that the material is both (weakly) elastically and (strongly) texturally anisotropic. This is presumably because the elastic anisotropy is small.

(ii) For the $\{0006\}$ measurements, some points are "missing" in the figures on the curves corresponding to $\phi=0$, since the influence of the texture is quite strong in this case, and for some $\phi$ values, there is no grain in diffracting position (see also Fig. 10). It is worth mentioning though that this situation is very often encountered in stress measurements, since the most studied materials in this context (steels and titanium alloys) are generally strongly textured (see e.g. the experimental diffracting volume fractions in [30], which are on the same order of magnitude as the ones calculated here). Due to the discrete description of the texture in the present case, the evaluation of the diffracting volume depends strongly on the selected spread (taken here equal to $5^{\circ}$, which is quite small), but a diffracting volume equal to $1 \%$ still corresponds here to 500 orientations.

(iii) As soon as $2^{\text {nd }}$ order stresses are created within the material, some non linearity appears clearly on both total strain $\left\langle\varepsilon_{\phi \psi}\right\rangle_{\Omega \text { cal. }}$ and $2^{\text {nd }}$ order strain $\left\langle\varepsilon_{\phi \psi}\right\rangle_{\Omega \text { cal. }}^{I I}$ profiles (see Points 2 to 4).

(iv) For point 4 - which is similar to a case where $1^{\text {st }}$ order stresses are classically determined the total strain profile deviates significantly from linearity for both peaks, and especially for $\phi=0$.

(v) We can also estimate from these curves, the quantitative influence of the $2^{\text {nd }}$ order term, whenever it is present; it is seen on all concerned curves that $\left\langle\varepsilon_{\phi \psi}\right\rangle_{\Omega \text { cal. }}^{I}$ and $\left\langle\varepsilon_{\phi \psi}\right\rangle_{\Omega \text { cal. }}^{I I}$ are of the same order of magnitude, although not always of the same sign; generally, the $2^{\text {nd }}$ order 
term is smaller than the $1^{\text {st }}$ order one, but it can sometimes be larger (for example, for point 4 , at $\phi=\psi=0$ for the $\{12 \overline{3} 3\}$ peak, we have $\left\langle\varepsilon_{\phi \psi}\right\rangle_{\Omega \text { cal. }}^{I I}=0.001$ and $\left.\left\langle\varepsilon_{\phi \psi}\right\rangle_{\Omega \text { cal. }}^{I}=-0.0005\right)$.

The total strains calculated for the $\{12 \overline{3} 3\}$ peak at $\phi=0$ are now replotted in Fig. 11 for the 4 points. The influence of the imposed macroscopic stress on the maximal strain value is quite clear: this maximal value is close to 0.002 for an imposed stress equal to $200 \mathrm{MPa}$ in the elastic range (after the establishment of plastic residual stresses (Point 4) or not (Point 1)), whereas it reaches 0.004 for an imposed stress close to $500 \mathrm{MPa}$ in the elasto-plastic range, and is reduced to 0.001 in the unloaded condition (which would correspond to the case of a macroscopic residual stress equal to 0$)$.

Now, considering that these calculated elastic strains are experimental data, we can estimate the $1^{\text {st }}$ order stresses for the 4 studied points through the minimization procedure explained above by neglecting the $2^{\text {nd }}$ order term $\left(1^{\text {st }}\right.$ assumption) and by assuming that the material is either (i) elastically isotropic (EI) (3rd assumption, Eq. 27), (ii) elastically anisotropic but macroscopically isotropic (IT) (Eq. 28) or (iii) elastically and macroscopically anisotropic (FA) (again Eq. 28). Two examples of this adjustment are shown in Fig. 12 and all resulting stress determinations are presented in Table 2.The stress state is also assumed to be plane stress, which is completely consistent with the imposed boundary conditions $\left(2^{\text {nd }}\right.$ assumption $)$. The minimization has been performed on points associated with one single peak (as in recommended standards) - here the $\{12 \overline{3} 3\}$ peak or the $\{0006\}$ peak, or with two peaks simultaneously (proposed method) (see Eq. 29).

The data presented in Fig. 12 correspond in a sense to two extreme cases. The $1^{\text {st }}$ one (Fig. 11 left) concerns the estimation of a sole $1^{\text {st }}$ order stress tensor in the absence of $2^{\text {nd }}$ order stresses. In this case, the adjustment is necessarily better than in all other cases, since the neglected term is 0 . Also, the influence of elastic or textural anisotropy is quite limited, since all curves are linear and the calculated error parameter is indeed quite small for all performed adjustments. The $2^{\text {nd }}$ case presented in Fig. 12 (right) corresponds to the worst case for stress estimation, since both terms are present in the predicted value. It is seen that the 4 calculated profiles are non linear in this case (see also Fig. 9). The adjusted profiles look however linear, in spite of the fact that the texture is taken into account. This is again presumably due to the weak elastic anisotropy.

Now, from the close inspection of the estimated stress values for each point and associated error parameter (Table 2), we can say that:

(i) For Point 1, for which the only origin of stress is elasticity, the adjustment is quite good for all cases, but always slightly better by taking into account both elastic and plastic anisotropy; the minimization on two peaks is also better than the minimization on one single peak; 
(ii) For Point 2, for which the level of stress is quite high, again taking into account both elastic and textural anisotropy improves the quality of the fits, except for the $\{0006\}$ one for which it has been seen that the diffracting volume was reduced for a lot of measurement points; minimizing on two peaks however still provides a better estimation than the minimization on one single peak;

(iii) Point 3 corresponds to the situation in which the pseudo-experimental elastic strain is composed on the sole term which is neglected in the minimization procedure. Whatever the selected peak, the error appears smaller when the elastic (and thus also textural) anisotropy is neglected.

(iv) For Point 4, taking into account the anisotropy of the material does not improve the quality of the fit, when the minimization is performed on one single peak. However, when the fit is performed simultaneously on two peaks, the quality of the fit is significantly improved, and especially when all sources of anisotropy are taken into account. In such a case, the error parameter is indeed divided by 1.12 when going from the recommended standard $\left(1^{\text {st }}\right.$ adjustment line, last column $\chi=124.9)$ to a double peak minimization ( $7^{\text {th }}$ adjustment line, last column $\chi=56.4)$.

It is thus clear from Table 2 that the adjustment on one single peak is acceptable only when there is no $2^{\text {nd }}$ order stress of inelastic origin (point 1 only) but also that in the 3 other cases, the adjustment is considerably improved by performing the minimization on both peaks simultaneously. This is nicely illustrated graphically in Fig. 13. The proposed multipeak minimization performed by taking into account both elastic anisotropy and texture (results highlighted in pink in Table 2) provides a much better estimation of the macroscopic stresses than the procedure recommended by actual standards (results highlighted in grey in Table 2) for the situations where $1^{\text {st }}$ and $2^{\text {nd }}$ order stresses are present. This is a very interesting result, since most of the cases for which stresses are estimated in practice correspond to cases for which both $1^{\text {st }}$ and $2^{\text {nd }}$ order terms (of various origins and not only of plastic origin) are present. It is also worth noting that although the calculated curves may appear quite close for Points 1 and 4 (see Fig. 11), the corresponding adjusted stress values resulting from a single peak minimization can be quite different (see Table 2).

\section{Conclusions and perspectives}

We have detailed in the present paper the classical procedure for the $1^{\text {st }}$ order stress estimation from XRD and calculated with a model the $1^{\text {st }}$ and $2^{\text {nd }}$ order stresses and associated elastic strains arising 
from an elasto-plastic deformation in a Ti tensile sample. The purpose of this calculation was not to estimate the predictive capacity of the model or to compare experimental and calculated data as in previous studies [30, 31], but simply to produce a virtual experimental data set. Thus, by considering the calculated data as pseudo-experimental data, we were able to apply on them the method recommended by all standards for stress determination, and to compare the results to a method based on multi-peak minimization.

For the tested case, we have shown that

- Neglecting the $2^{\text {nd }}$ order stresses in the determination of the $1^{\text {st }}$ order ones induces a significant error when the standard method is employed (i.e. minimization on one single peak); this point has already been underlined by several authors, which compared in the past experimental and calculated profiles [30, 31]. However, these authors did not quantify the error made by completely neglecting the $2^{\text {nd }}$ order term as in the present case (aimed at evaluating the capacity of the proposed minimization procedure to estimate the $1^{\text {st }}$ order stress without being obliged to calculate the $2^{\text {nd }}$ order term), and introduced rather an additional adjusting parameter to minimize the error made between experimental and calculated profiles [15], and thus to evaluate the predicting capacity of one model in simple cases. The use of an additional adjusting parameter has also been employed by other authors more recently $[14,32]$.

- This error is considerably reduced when minimization is performed on two peaks, although the reason for this improvement is not completely clear yet;

- When minimization is performed on two peaks, taking into account elastic anisotropy (IT or FA hypotheses) and texture only very slightly improves the result compared to the IE hypothesis.

As the presented data obviously depend on the selected material (texture) and sampling procedure, this work is now continued to test the validity of the multi-peak minimization in other cases, that is for different texture and different stress origins (and especially with residual stresses due to thermal origin or phase transformation), in single and dual phase Ti alloys. Also, some experimental data obtained with the new portable device $[26,28]$ are presently analyzed to confirm the validity of a multi-peak analysis. Indeed, with this device, it is possible to measure elastic strains during a tensile test (in order to know a priori the value of the $1^{\text {st }}$ order macroscopic stress, just like in the present work), Also, the analysis of the data, which is performed with the software MAUD ${ }^{\S}$ is based on Rietveld refinement, i.e. based on a multi-peak minimization.

${ }^{\S}$ http://www.ing.unitn.it/ maud/beta.html 
Acknowledgments: Fruitful discussions about residual stresses with D. Aliaga and N. Guillemot from Airbus Helicopters are acknowledged.

Funding: The authors would like to thank the Conseil Général de Seine St Denis (CG93) for the attribution of a $\mathrm{PhD}$ fellowship to S. Dufrenoy.

Conflict of Interest: The authors declare that they have no conflict of interest.

\section{References}

1. Withers, P.J., Residual stress and its role in failure. Reports on Progress in Physics, 2007. 70(12): p. 2211.

2. Bretheau, T. and O. Castelnau, Les contraintes résiduelles : d'où viennent-elles ? Comment les caractériser? , in Rayons X et Matière. 2006, Lavoisier. p. 123-153.

3. Noyan, I.C. and J.B. Cohen, An X-ray diffraction study of the residual stress-strain distributions in shot-peened two-phase brass. Materials Science and Engineering, 1985. 75(1): p. 179-193.

4. Turner, P.A. and C.N. Tomé, A study of residual stresses in Zircaloy-2 with rod texture. Acta Metallurgica Et Materialia, 1994. 42(12): p. 4143-4153.

5. Baczmański, A., N. Hfaiedh, M. François, and K. Wierzbanowski, Plastic incompatibility stresses and stored elastic energy in plastically deformed copper. Materials Science and Engineering: A, 2009. 501(1-2): p. 153-165.

6. Turner, P.A., N. Christodoulou, and C.N. Tomé, Modeling the mechanical response of rolled Zircaloy-2. International Journal of Plasticity, 1995. 11(3): p. 251-265.

7. Afnor, J., Non-destructive Testing — Test Method for Residual Stress analysis by X-ray Diffraction, AFNOR.

8. Pang, J.W.L., T.M. Holden, and T.E. Mason, In situ generation of intergranular strains in an Al7050 alloy. Acta Materialia, 1998. 46(5): p. 1503-1518.

9. Clausen, B., T. Lorentzen, M.A.M. Bourke, and M.R. Daymond, Lattice strain evolution during uniaxial tensile loading of stainless steel. Materials Science and Engineering: A, 1999. 259(1): p. 17-24.

10. Zhu, K.Y., B. Bacroix, T. Chauveau, D. Chaubet, and O. Castelnau, Texture Evolution and Associated Nucleation and Growth Mechanisms during Annealing of a $\mathrm{Zr}$ Alloy. Metallurgical and Materials Transactions a-Physical Metallurgy and Materials Science, 2009. 40A(10): p. 2423-2434.

11. Kroner, E., Berechnung der Elastischen Konstanten des Vielkristalls aus den Konstanten des Einkristalls. Zeitschrift für Physik, 1958. 151(4): p. 504-518.

12. Hutchinson, J., Elastic-plastic behaviour of polycrystalline metals and composites. Proceedings of the Royal Society of London Series a-Mathematical and Physical Sciences, 1970. 319(1537): p. 247-\&.

13. Tome, C.N., N. Christodoulou, P.A. Turner, M.A. Miller, C.H. Woo, J. Root, and T.M. Holden, Role of internal stresses in the transient of irradiation growth of Zircaloy-2. Journal of Nuclear Materials, 1996. 227(3): p. 237-250.

14. Gloaguen, D., T. Berchi, E. Girard, and R. Guillén, Measurement and prediction of residual stresses and crystallographic texture development in rolled Zircaloy-4 plates: X-ray diffraction and the self-consistent model. Acta Materialia, 2007. 55(13): p. 4369-4379. 
15. Baczmański, A., K. Wierzbanowski, P. Lipiński, R.B. Helmholdt, G. Ekambaranathan, and B. Pathiraj, Examination of the residual stress field in plastically deformed polycrystalline material. Philosophical Magazine A, 1994. 69(3): p. 437-449.

16. Jeong, G.S., D.H. Allen, and D.C. Lagoudas, Residual stress evolution due to cool down in viscoplastic metal matrix composites. International Journal of Solids and Structures, 1994. 31(19): p. 2653-2677.

17. Turner, P.A. and C.N. Tomé, Self-consistent modeling of visco-elastic polycrystals: Application to irradiation creep and growth. Journal of the Mechanics and Physics of Solids, 1993. 41(7): p. 1191-1211.

18. Zecevic, M., M. Knezevic, I.J. Beyerlein, and C.N. Tomé, An elasto-plastic self-consistent model with hardening based on dislocation density, twinning and de-twinning: Application to strain path changes in HCP metals. Materials Science and Engineering: A, 2015. 638: p. 262-274.

19. Yoshida, K., R. Brenner, B. Bacroix, and S. Bouvier, Micromechanical modeling of the workhardening behavior of single- and dual-phase steels under two-stage loading paths. Materials Science and Engineering a-Structural Materials Properties Microstructure and Processing, 2011. 528(3): p. 1037-1046.

20. Benmhenni, N., S. Bouvier, R. Brenner, T. Chauveau, and B. Bacroix, Micromechanical modelling of monotonic loading of CP alpha-Ti: Correlation between macroscopic and microscopic behaviour. Materials Science and Engineering a-Structural Materials Properties Microstructure and Processing, 2013. 573: p. 222-233.

21. Fisher, E.S. and C.J. REnken, Single-crystal elastic moduli and the hcp -> bcc transformation in Ti; $\mathrm{Zr}$ and $H f$. Physical Review, 1964. 165(2A): p. 482-500.

22. Guillemot, N., M. Winter, A. Souto-Lebel, C. Lartigue, and R. Billardon, 3D Heat Transfer Analysis for a Hybrid Approach to Predict Residual Stresses After Ball-End Milling. Procedia Engineering, 2011. 19: p. 125-131.

23. Webster, G.A. and R.C. Wimpory, Non-destructive measurement of residual stress by neutron diffraction. Journal of Materials Processing Technology, 2001. 117(3): p. 395-399.

24. Daymond, M.R. and P.J. Withers, A new stroboscopic neutron diffraction method for monitoring materials subjected to cyclic loads: Thermal cycling of metal matrix composites. Scripta Materialia, 1996. 35(6): p. 717-720.

25. Ligot, J., U. Welzel, P. Lamparter, A.C. Vermeulen, and E.J. Mittemeijer, Stress analysis of polycrystalline thin films and surface regions by X-ray diffraction. Journal of Applied Crystallography, 2005. 38(1): p. 1-29.

26. Dufrenoy, S., B. Bacroix, C. Th., I. Lemaire, N. Guillemot, and G. Thomas. Influence of surface integrity on fatigue limit: Ti-10V-2Fe-3Al titanium application. in 13th World Conference on Titanium. 2015. San Diego, USA: TMS.

27. Belkhabbaz, A., R. Brenner, N. Rupin, B. Bacroix, and J. Fonseca, Prediction of the overall behavior of a 3D microstructure of austenitic steel by using FFT numerical scheme, in 11th International Conference on the Mechanical Behavior of Materials, M. Guagliano and L. Vergani, Editors. 2011. p. 1883-1888.

28. Dufrenoy, S., T. Chauveau, R. Brenner, C. Fontugne, and B. Bacroix, Modeling methodology for stress determination by XRD in polycrystalline materials. Residual Stresses Ix, 2014. 996: p. 106-111.

29. Daymond, M.R., C.N. Tome, and M.A.M. Bourke, Measured and predicted intergranular strains in textured austenitic steel. Acta Materialia, 2000. 48(2): p. 553-564.

30. Jeong, Y., T. Gnäupel-Herold, F. Barlat, M. Iadicola, A. Creuziger, and M.-G. Lee, Evaluation of biaxial flow stress based on elasto-viscoplastic self-consistent analysis of X-ray diffraction measurements. International Journal of Plasticity, 2015. 66: p. 103-118.

31. Baczmański, A. and C. Braham, Elastoplastic properties of duplex steel determined using neutron diffraction and self-consistent model. Acta Materialia, 2004. 52(5): p. 1133-1142. 
32. Gloaguen, D., J. Fajoui, and B. Girault, Residual stress fields analysis in rolled Zircaloy-4 plates: Grazing incidence diffraction and elastoplastic self-consistent model. Acta Materialia, 2014. 71: p. 136-144. 


\begin{tabular}{|c|c|c|}
\hline $\begin{array}{c}\text { Slip family (number of } \\
\text { systems) }\end{array}$ & $\tau_{c}^{S}(\mathrm{MPa})$ & $q=1.003$ \\
\cline { 1 - 2 }$\{1 \overline{1} 00\}\langle 1 \overline{2} 0\rangle(3)$ & 105 & $h_{0}=81300 \mathrm{MPa}$ \\
& & $n=0.047$ \\
\cline { 1 - 2 }$\{0001\}\langle 11 \overline{2} 0\rangle(3)$ & 125 & $\tau_{\text {ref }}=105 \mathrm{MPa}$ \\
\hline$\{10 \overline{1} 1\}\langle 11 \overline{2} 3\rangle(12)$ & 215 & \\
\hline
\end{tabular}

Table 1: Selected slip systems for titanium and associated hardening parameters. 


\begin{tabular}{|c|c|c|c|c|c|c|c|c|}
\hline & \multicolumn{3}{|c|}{ Point 1 (elastic loading) } & \multirow[b]{2}{*}{$\chi$} & \multicolumn{3}{|c|}{ Point 2 (elasto-plastic loading) } & \multirow[b]{2}{*}{$\chi$} \\
\hline & $\sigma_{11}$ & $\sigma_{22}$ & $\sigma_{12}$ & & $\sigma_{11}$ & $\sigma_{22}$ & $\sigma_{12}$ & \\
\hline Calculated & 200 & $\mathbf{0}$ & $\mathbf{0}$ & & 478 & $\mathbf{0}$ & $\mathbf{0}$ & \\
\hline \multicolumn{9}{|c|}{$12 \overline{3} 3$} peak \\
\hline Adjust. IE & 213 & -8 & 2 & 15.4 & 433 & -115 & -15 & 124.4 \\
\hline Adjust. IT & 215 & -7 & 2 & 16.7 & 439 & -117 & -15 & 124.2 \\
\hline Adjust. FA & 211 & 3 & 2 & 11.6 & 424 & -97 & -14 & 111.9 \\
\hline \multicolumn{9}{|c|}{0006} peak \\
\hline Adjust. IE & 178 & -7 & 0 & 23.1 & 556 & -9 & 58 & 62.7 \\
\hline Adjust. IT & 203 & -8 & 1 & 8.6 & 633 & -10 & 66 & 168.0 \\
\hline Adjust. FA & 198 & 0 & 1 & 2 & 619 & 16 & 63 & 155.0 \\
\hline $\multicolumn{9}{|c|}{12 \overline{3} 3}+\{0006\}$ peaks \\
\hline Adjust. IE & 210 & -8 & 0 & 12.8 & 494 & -62 & 13 & 63.3 \\
\hline Adjust. IT & 214 & -8 & -1 & 16.5 & 509 & -70 & 13 & 77.6 \\
\hline Adjust. FA & 209 & 3 & -2 & 9.7 & 493 & -44 & 11 & 47.8 \\
\hline
\end{tabular}

\begin{tabular}{|c|c|c|c|c|c|c|c|c|}
\hline & \multicolumn{3}{|c|}{ Point 3 (unloading) } & \multirow[b]{2}{*}{$\chi$} & \multicolumn{3}{|c|}{ Point 4 (elastic reloading) } & \multirow[b]{2}{*}{$\chi$} \\
\hline & $\sigma_{11}$ & $\sigma_{22}$ & $\sigma_{12}$ & & $\sigma_{11}$ & $\sigma_{22}$ & $\sigma_{12}$ & \\
\hline Calculated & $\mathbf{0}$ & $\mathbf{0}$ & $\mathbf{0}$ & & 200 & $\mathbf{0}$ & $\mathbf{0}$ & \\
\hline \multicolumn{9}{|c|}{$12 \overline{3} 3$} peak \\
\hline Mod. IE & -79 & -98 & -17 & 127.0 & 134 & -105 & -15 & 124.9 \\
\hline Mod. IT & -80 & -99 & -17 & 128.4 & 135 & -106 & -15 & 125.2 \\
\hline Mod. FA & -87 & -105 & -16 & 137.3 & 125 & -101 & -14 & 126.6 \\
\hline \multicolumn{9}{|c|}{0006} peak \\
\hline Mod. IE & 126 & 7 & 21 & 127.9 & 305 & 0 & 22 & 107.3 \\
\hline Mod. IT & 144 & 9 & 25 & 146.4 & 346 & 0 & 26 & 148.3 \\
\hline Mod. FA & 141 & 15 & 24 & 143.8 & 338 & 14 & 25 & 140.9 \\
\hline $\multicolumn{9}{|c|}{12 \overline{3} 3}+\{0006\}$ peaks \\
\hline Mod. IE & 7 & -48 & 2 & 48.5 & 209 & -56 & 2 & 56.7 \\
\hline Mod. IT & -9 & -58 & 5 & 58.9 & 205 & -66 & 4 & 66.3 \\
\hline Mod. FA & -15 & -59 & 6 & 61.2 & 195 & -56 & 4 & 56.4 \\
\hline
\end{tabular}

Table 2: Comparison of calculated and adjusted principal stresses for the 4 investigated points and 3 different assumptions concerning the material. The least square procedure has been applied on $\{12 \overline{3} 3\}$ and $\{0006\}$ peaks, first separately and then together. The calculated error parameter $\chi$ is equal to:

$$
\chi=\sqrt{\left(\sigma_{11 \text { cal. }}-\sigma_{11 \text { mod. }}\right)^{2}+\left(\sigma_{22 \text { cal. }}-\sigma_{22 \text { mod. }}\right)^{2}+\left(\sigma_{12 \text { cal. }}-\sigma_{12 \text { mod. }}\right)^{2}} .
$$




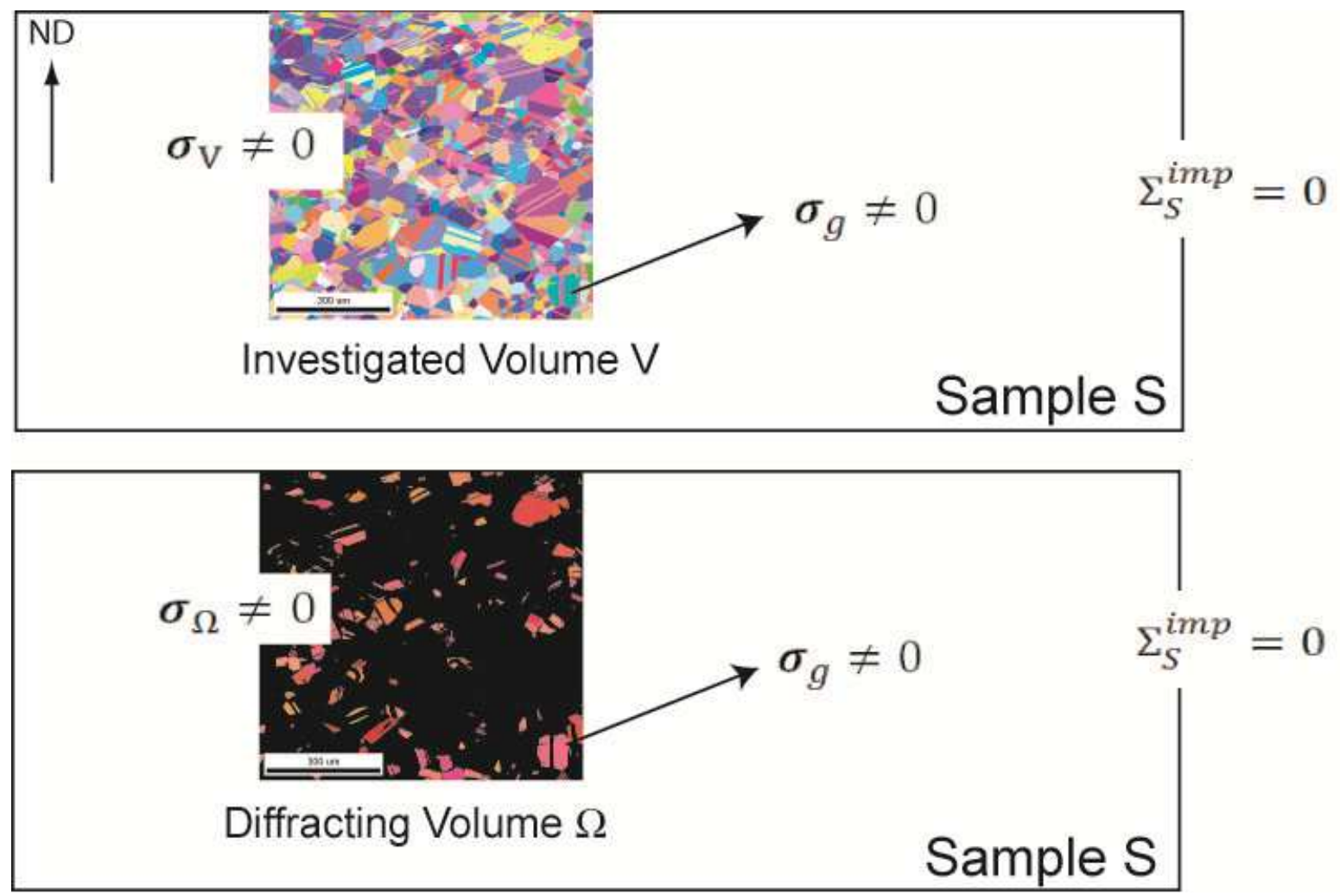

Fig. 1: Definition of the investigated $\mathbf{V}$ and diffracting $\Omega$ volumes. Illustration of the $1^{\text {st }}$ and $2^{\text {nd }}$ order stresses in an unloaded sample.
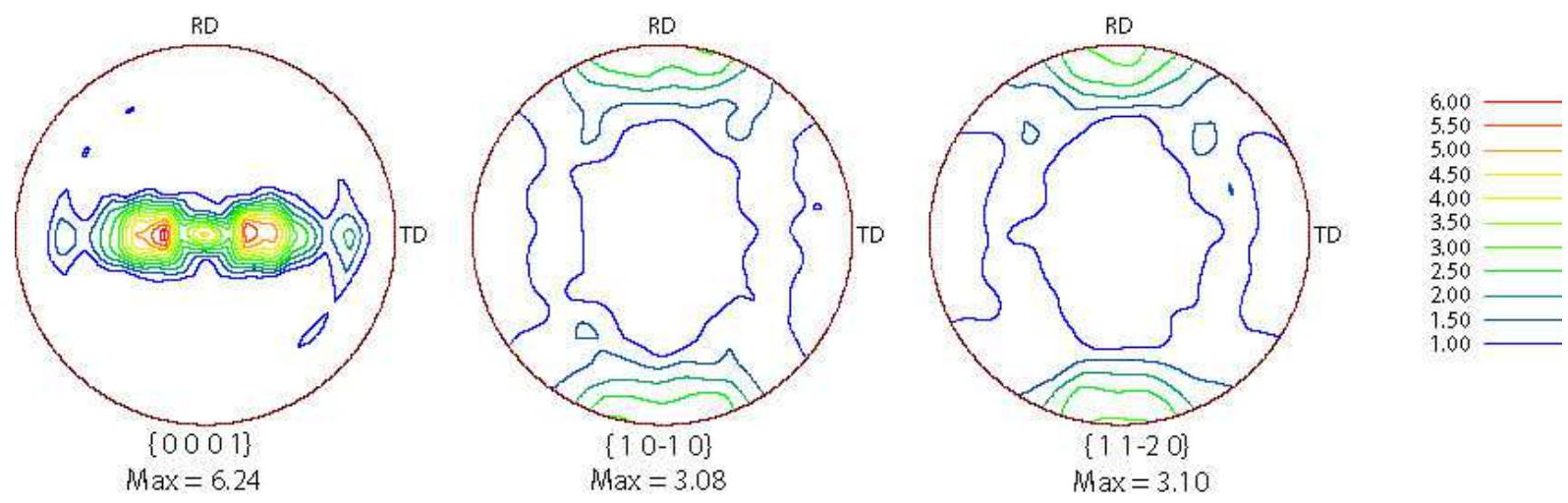

Fig. 2: Pole figures measured by X-ray Diffraction on a rolled and annealed titanium sample. RD and TD stand for rolling and transverse directions respectively. The intensities are multiples of the random intensity. 

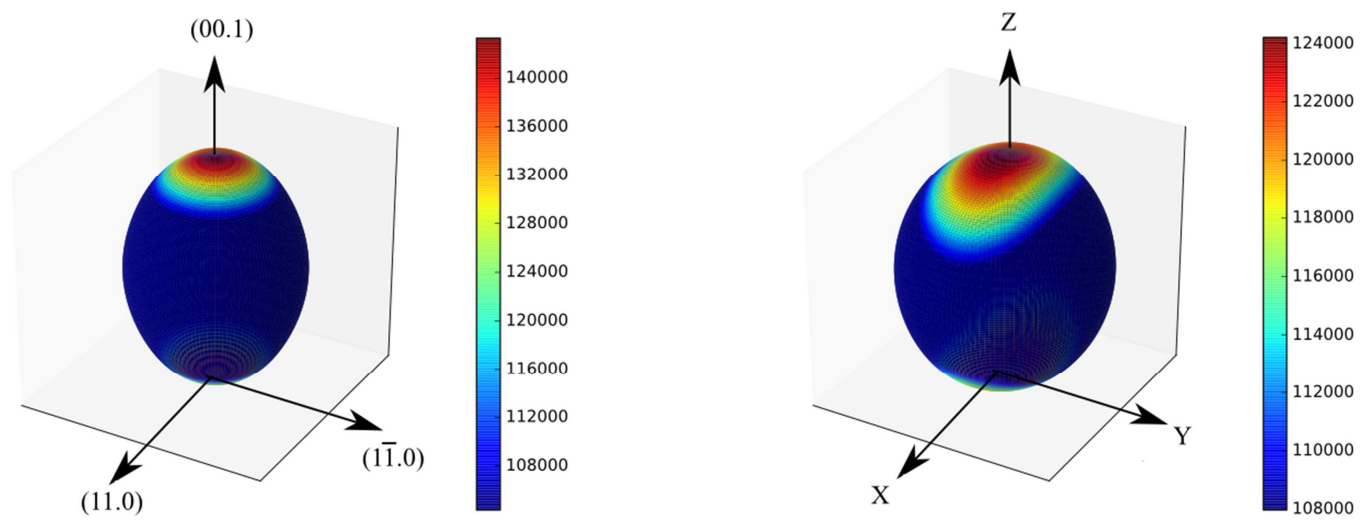

Fig. 3. Calculated Young's modulus for a Ti HCP crystal expressed in the crystal frame (left) and for the T40 sample expressed in the macroscopic frame (right). The values are expressed in GPa.
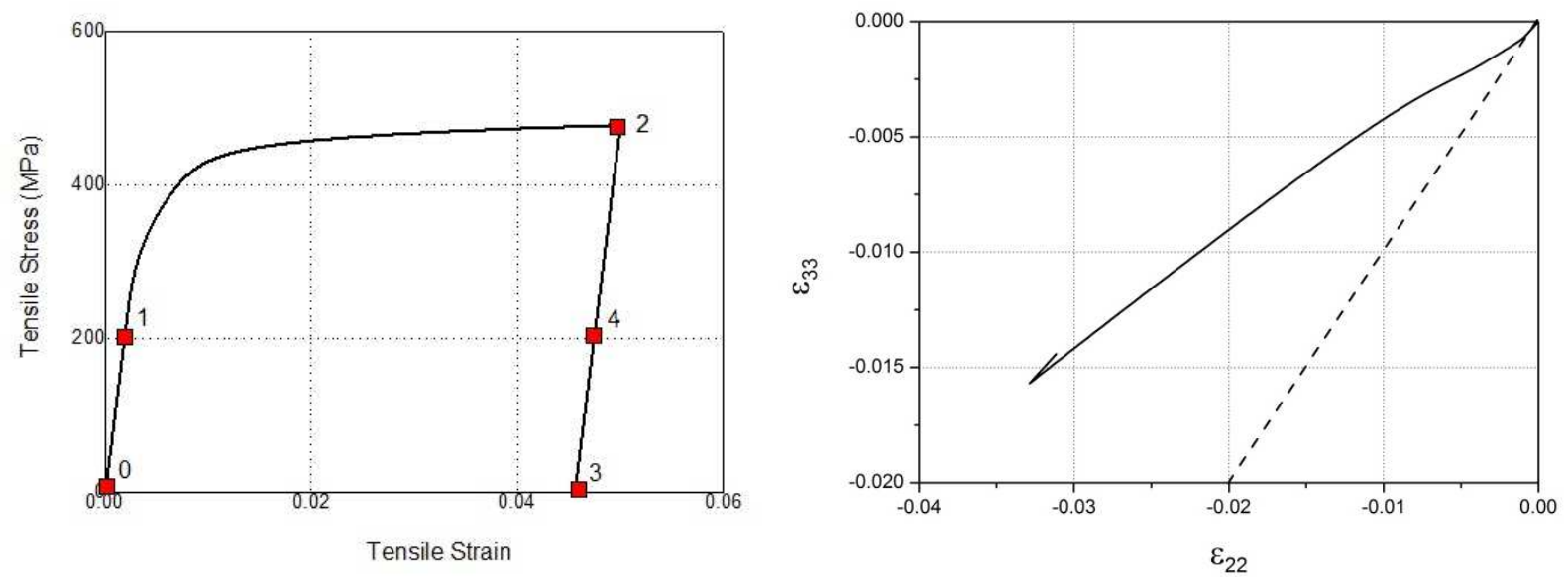

Fig. 4. Simulated tensile curve for a textured titanium sample (left) and Evolution of the transverse and thickness strains (the dashed line corresponds to $\mathrm{y}=\mathrm{x}$ ) (right). 


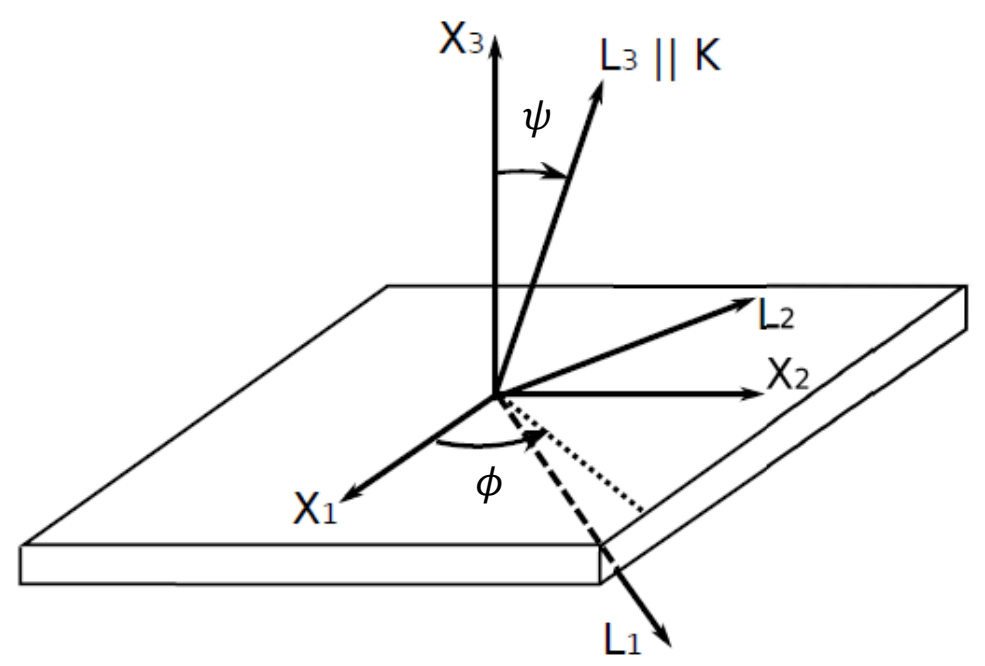

Fig. 5: Definition of the two reference frames, linked to the sample (X1, X2, X3) and to the diffracting vector, characterized by the two angles $\psi$ and $\phi$ and the axes (L1, L2, L3).

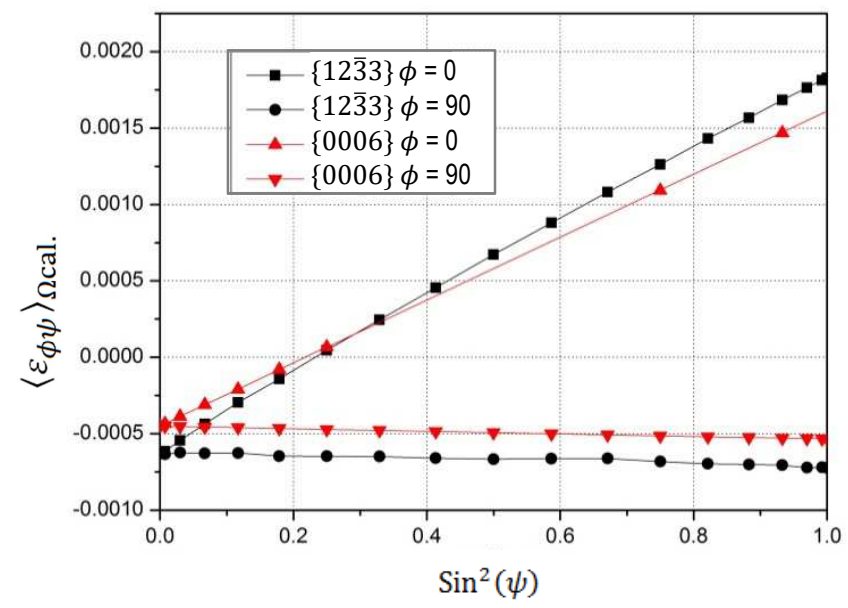

Fig. 6: Calculated elastic strains $\left\langle\varepsilon_{\phi \psi}\right\rangle_{\Omega \text { cal. }}$ as a function of $\sin ^{2} \psi$ for Point 1 (elastic loading) on the tensile curve; the macroscopic elastic strain depends only on the macroscopic $1^{\text {st }}$ order stress tensor:

$$
\left\langle\varepsilon_{\phi \psi}\right\rangle_{\Omega \text { cal. }}=\left\langle\varepsilon_{\phi \psi}\right\rangle_{\Omega \text { cal. }}^{I}
$$



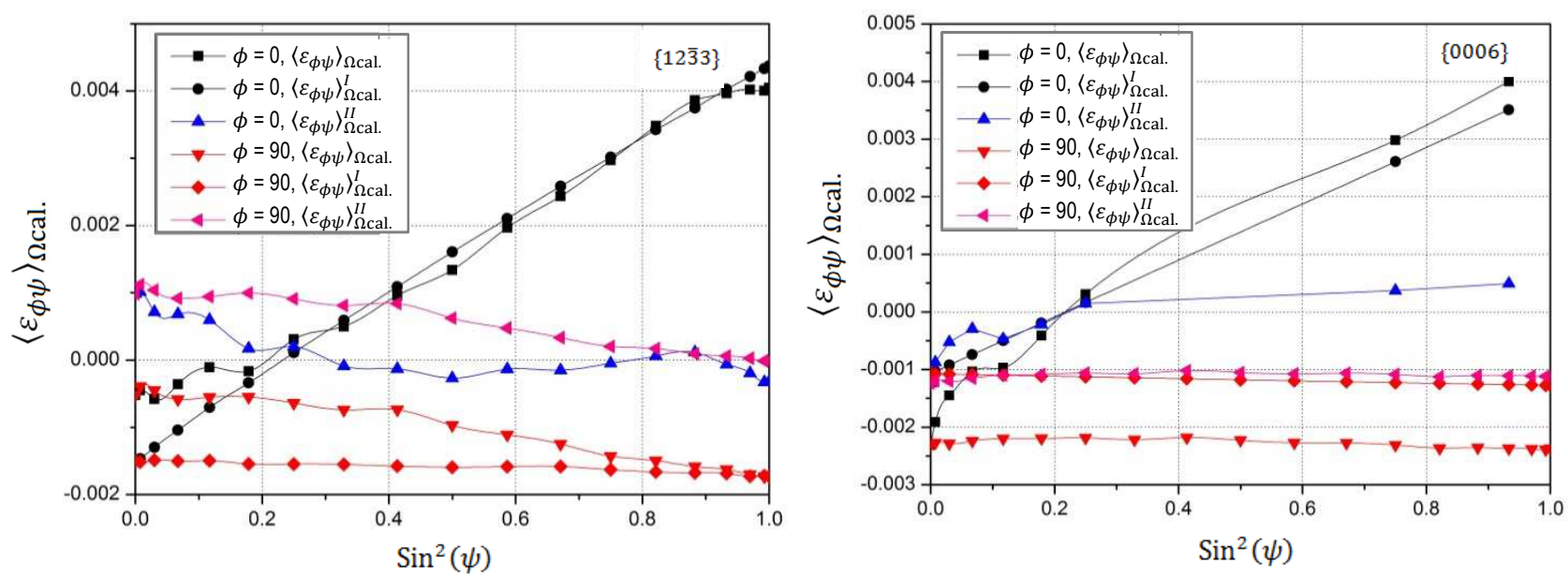

Fig. 7: Calculated elastic strains $\left\langle\varepsilon_{\phi \psi}\right\rangle_{\Omega \text { cal. }}$ as a function of $\sin ^{2} \psi$ for Point 2 (elastoplastic loading) on the tensile curve; the macroscopic elastic strain depends on $1^{\text {st }}$ and $2^{\text {nd }}$ order stress tensors:

$$
\left\langle\varepsilon_{\phi \psi}\right\rangle_{\Omega \text { cal. }}=\left\langle\varepsilon_{\phi \psi}\right\rangle_{\Omega \text { cal. }}^{I}+\left\langle\varepsilon_{\phi \psi}\right\rangle_{\Omega \text { cal. }}^{I I} .
$$

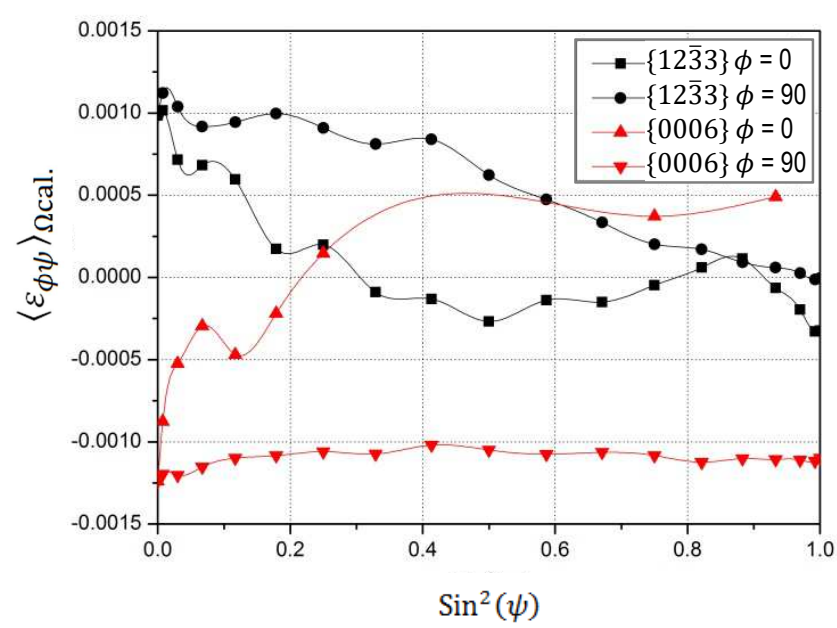

Fig. 8: Calculated elastic strains $\left\langle\varepsilon_{\phi \psi}\right\rangle_{\Omega \text { cal. }}$ as a function of $\sin ^{2} \psi$ for Point 3 (unloading after elastoplastic loading) on the tensile curve; the macroscopic elastic strain depends only on the residual $2^{\text {nd }}$ order stress tensor: $\left\langle\varepsilon_{\phi \psi}\right\rangle_{\Omega \text { cal. }}=\left\langle\varepsilon_{\phi \psi}\right\rangle_{\Omega \text { cal. }}^{I I}$. 

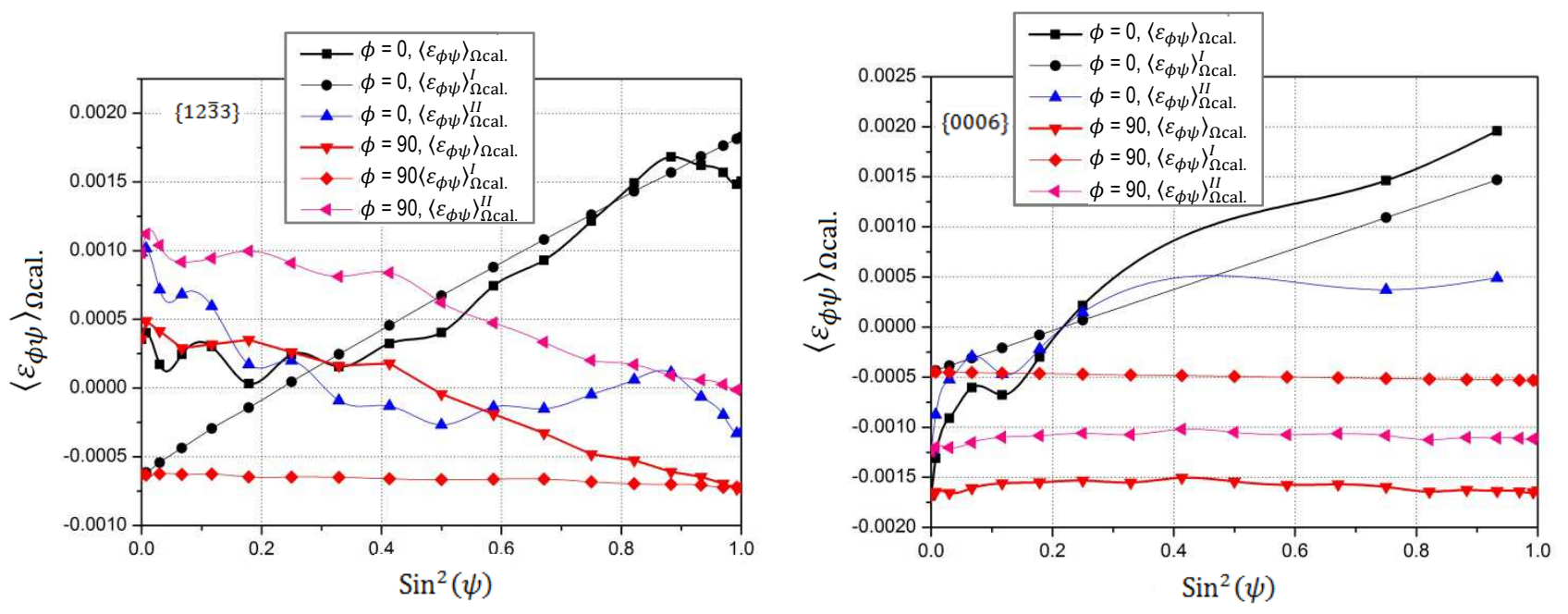

Fig. 9: Calculated elastic strains $\left\langle\varepsilon_{\phi \psi}\right\rangle_{\Omega \text { cal. }}$ as a function of $\sin ^{2} \psi$ for Point 4 (elastic reloading after elastoplastic loading and unloading) on the tensile curve; the macroscopic elastic strain depends again on both $1^{\text {st }}$ and $2^{\text {nd }}$ order stress tensors; $\left\langle\varepsilon_{\phi \psi}\right\rangle_{\Omega \text { cal. }}=\left\langle\varepsilon_{\phi \psi}\right\rangle_{\Omega \text { cal. }}^{I}+\left\langle\varepsilon_{\phi \psi}\right\rangle_{\Omega \text { cal. }}^{I I}$.

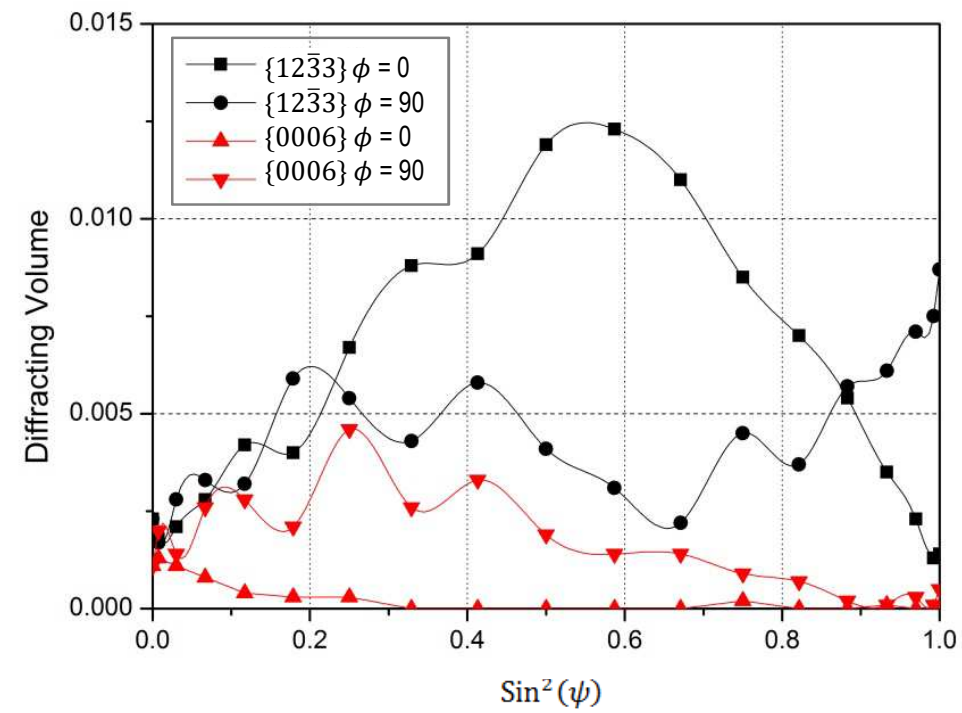

Fig. 10: Calculated diffracting volumes as a function of $\sin ^{2} \psi$ for the two investigated profiles. 


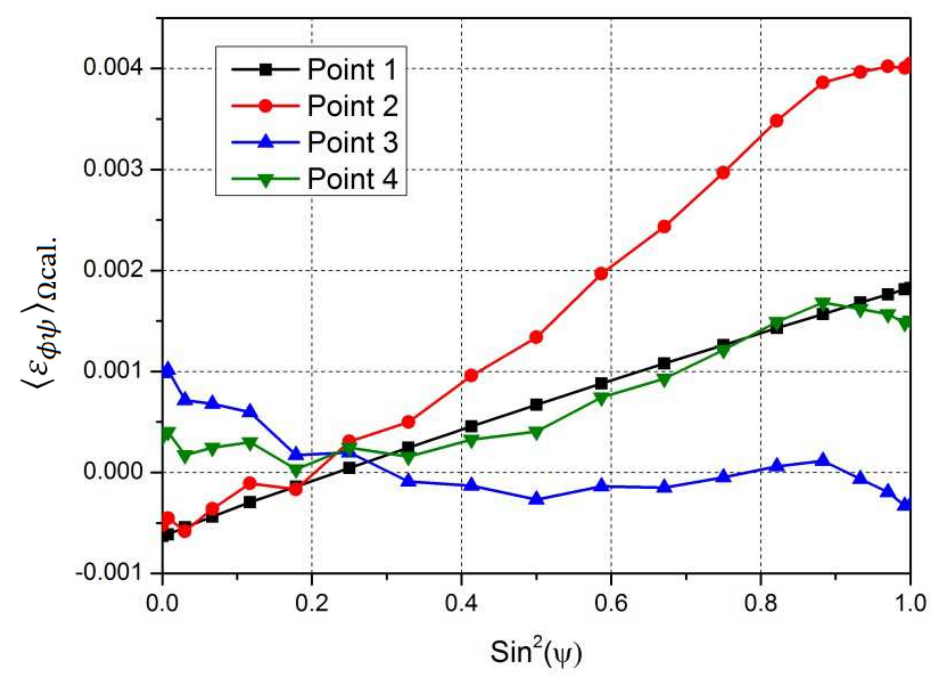

Fig. 11: Calculated elastic strains $\left\langle\varepsilon_{\phi \psi}\right\rangle_{\Omega \text { cal. }}$ as a function of $\sin ^{2} \psi$ for the four investigated points.

$\{12 \overline{3} 3\}$ peak, $\phi=0$.
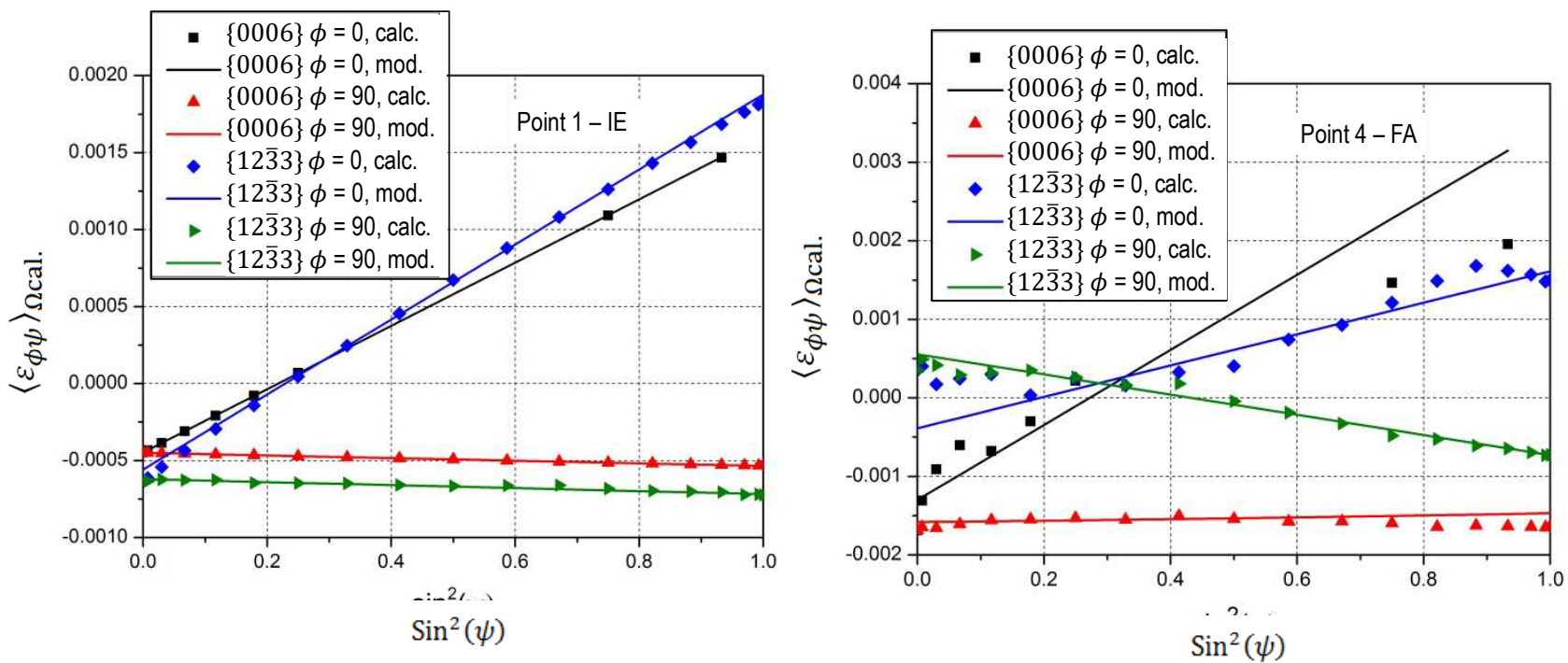

Fig. 12: Comparison of pseudo-experimental (calc.) and adjusted (mod.) elastic strains through the least square procedure given by Eq. (29) for: (left) point 1 (elastic loading), assumption of isotropic elasticity and (right) point 4 (elastic loading after elasto-plastic loading and unloading), assumption of anisotropic elasticity and texture. 


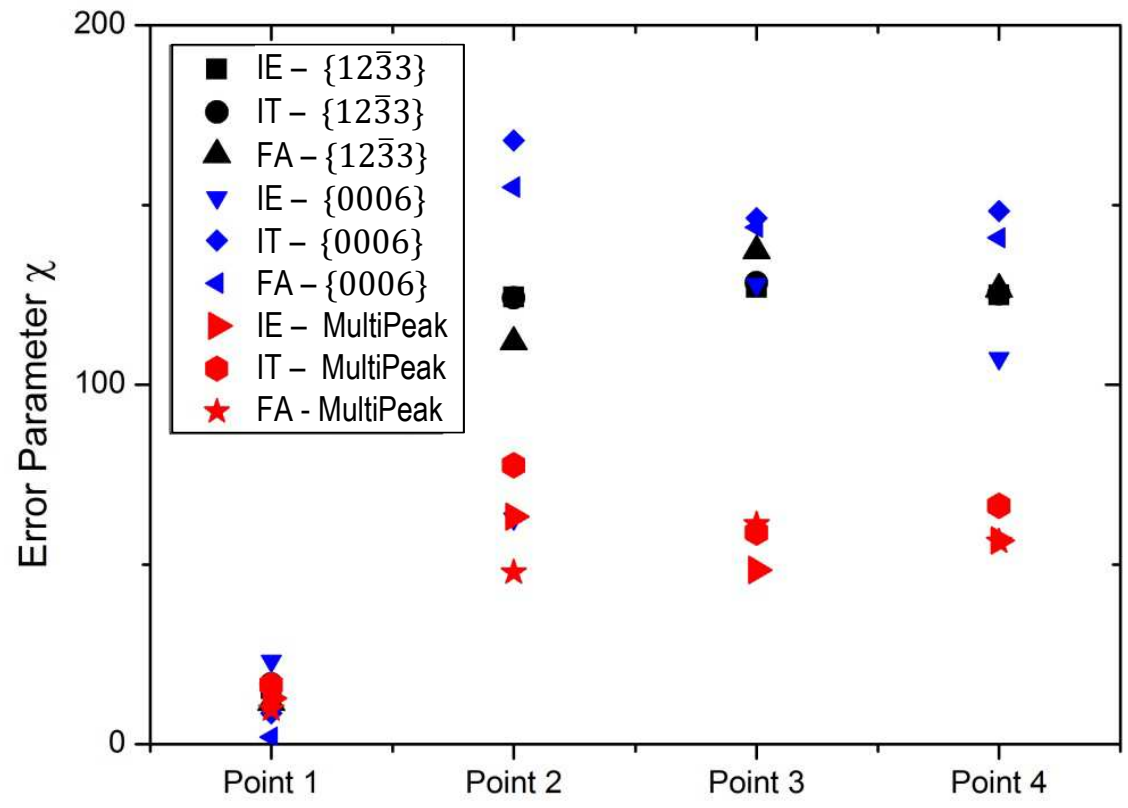

Fig. 13: Representation of the error parameter for the various adjustment procedures for all investigated points. 